$34-72$

\title{
RECORDS OF WELLS AND WATER QUALITY FOR THE GARBER-WELLINGTON AQUIFER, NORTHERN OKLAHOMA AND SOUTHERN LOGAN COUNTIES, OKLAHOMA
}

U. S. GEOLOGICAL SURVEY

Open-File Report 76-619

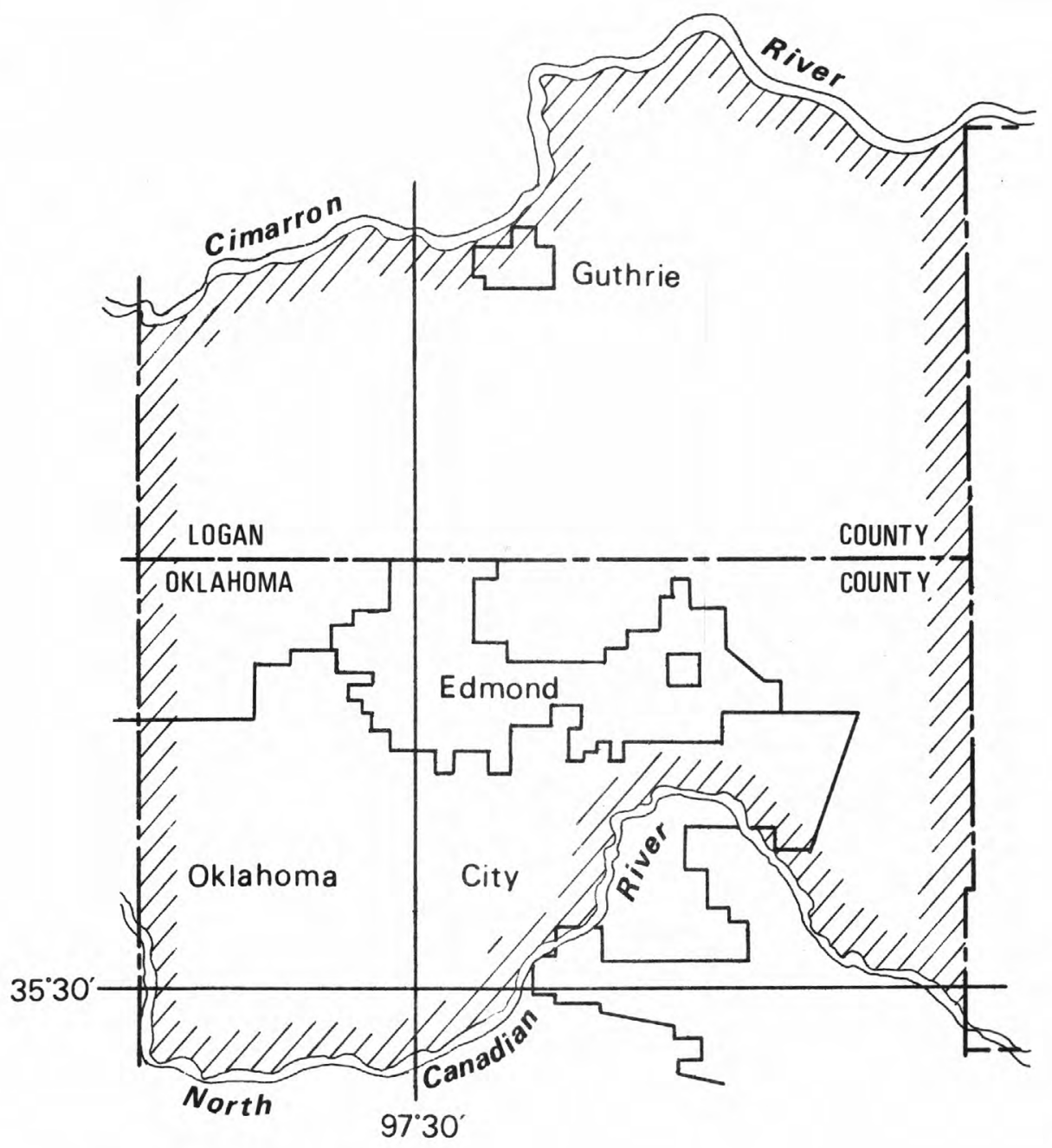

Prepared in cooperation with

U.S. BUREAU OF RECLAMATION

and

OKLAHOMA GEOLOGICAL SURVEY 



\section{UNITED STATES \\ DEPARTMENT OF THE INTERIOR \\ Geological Survey}

RECORDS OF WELLS AND WATER QUALITY FOR THE GARBER-WELLINGTON AQUIFER,

NORTHERN OKLAHOMA AND

SOUTHERN LOGAN COUNTIES, OKLAHOMA

Compiled by Jerry E. Carr and John S. Havens

0pen-File Report 76-619

Prepared in cooperation with

U. S. BUREAU OF RECLAMATION

and

OKLAHOMA GEOLOGICAL SURVEY

Oklahoma City, Oklahoma

September, 1976 



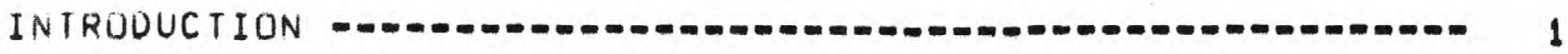

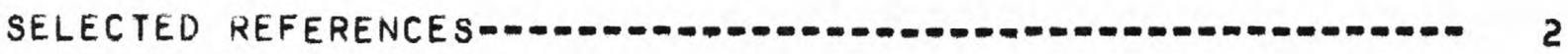

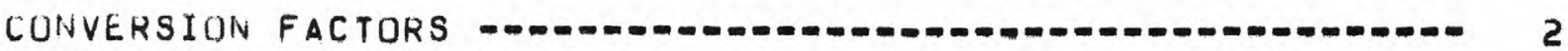

EXPLANATIUN UF INFORMATION IN TABLE 1

EXPLANATIUN OF INFORMATIUN IN TABLE 2

\section{TABLES}

TABLE 1.--RECORDS OF WELLS IN THE GARBER-WELLINGTON

AQUIFER

UKLAHOMA CUUNTY

LOGAN COUNTY

TABLE 2. =-CHEMICAL ANALYSES OF GROUND WATER FRUM THE

GARBER-WELLINGTON AQUIFER

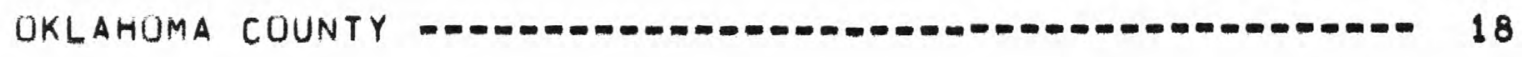

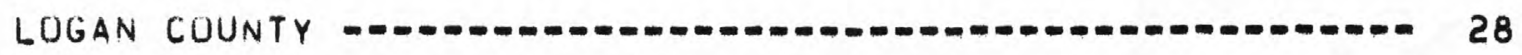





\begin{abstract}
RECORDS OF WELLS ANU WATER QUALITY FOR THE GARBERWELLINGTON AQUIFER, NORTHERN OKLAHOMA AND SOUTHERN LOGAN COUNTIES, OKLAHOMA
\end{abstract}

BY JERRY E. CARR AND JOHN S. HAVENS

INTRODUCTION

THE U. S. GEOLOGICAL SURVEY HAS COLLECTED DATA ON OKLAHOMA'S GROUND-WATER RESOURCES SINCE 1934. MOST OF THESE DATA WERE COLLECTED AS PART OF SPECIFIC GROUND-WATER STUDIES CONDUCTED IN COOPERATION WITH VARIOUS FEDERAL, STATE, ANO LOCAL AGENCIES.

IN 1972, AT THE REQUEST OF THE U. S, BUREAU OF RECLAMRTIUN, THE GEOLOGICAL SURVEY UNDERTOOK A STUOY OF THE GARBEKWELLINGTON AQUIFER IN NORTHERN OKLAHOMA ANO SOUTHERN LOGAN COUNTIES. AS A PART DF THIS PROJECT, RECORUS OF ABOUT 120 WELLS WERE COLLECTED AND 12 SAMPLES OF GROUND WATER FRC $~$ T THE AQUIFER WERE ANALYZED IN THE LABORATORIES OF THE GEOLOG:CAL SURVEY; THESE RECORDS ARE INCLUDED IN THIS REPORT. IN ADCITIUN, RECORDS OF ABOUT 302 WELLS AND ANALYSES OF 60 WATER SAMPLES WERE TAKEN FROM A REPORT BY WOOD AND BURTON(1960) AND FROM THE FILES OF THE GEOLOGICAL SURVEY.

ALSO INCLUDED IN THIS REPORT ARE SELECTED REFERENCES FUR THOSE DESIRING MORE INFORMATION ON THE AREA'S GROUND -WATER RESOURCES.

THE STRATIGRAPHIC NOMENCLATURE AND AGE DETERMINATIUNS USED IN THIS REPORT ARE THOSE ACCEPTED BY THE OKLAHOMA GEOLOGICAL SURVEY AND DO NUT NECESSARILY AGREE WITH THOSE OF THE U. S. GEOLOGICAL SURVEY.

ACKNOWLEDGMENT IS EXTENDED TO THE MANY HUNDREDS OF INDIVIDUALS WHO HAVE PROVIDED THE DATA COMPILED IN THIS REPORT. 
BINGHAM, R. H. AND MOURE, R. L. , 1975, RECONNAISSANCE OF THE WATER RESOURCES OF THE OKLAHOMA CITY QUADRANGLE, CENTRAL OKLAHOMA: OKLAHOMA GEOL. SURVEY HYOROL. ATLAS 4, 4 SHEETS, SCALE 1:250,000.

OKLAHUMA WATER RESOURCES BOARO, 1971, APPRAISAL DF THE WATER ANO RELATED LAND RESOURCES OF OKLAHOMA REGION EIGHT: OKLAMOMA WATER RESOURCES BOARD PUBLICATION $34,141 \mathrm{P}$.

PHELPS, SFITZ, AMMERMAN \& THOMAS, INC., 1975, REGIONAL WATER PLAN FOR CENTRAL OKLAHOMA: REPORT PREPARED FOR THE ASSOCI ATIUN OF CENTRAL OKLAHOMA GOVERNMENTS, OKLAHOMA CITY, $141 \mathrm{P}$. WOOD, P. R. ANO BURTON, L. C., 1968, GROUND-WATER RESOURCES IN CLEVELAND AND OKLAHOMA COUNTIES, OKLAHOMA: OKLAHOMA GEOL. SURVEY CIRD. $71,75 \mathrm{P}$.

\section{CONVERSION FACTORS}

ENGLISH UNITS USED IN THIS REPORT MAY BE CONVERTED TO METRIC UNITS BY THE FULLUWING CONVERSION FACTORS:

MULTIPLY ENGLISH UNIT BY TO OBTAIN METRIC UNIT

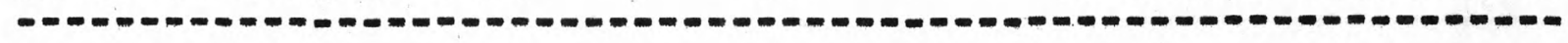

INCHES (IN)

FEET ( $F T$ )

GALLONS PER MINUTE (GAL/MIN)
25.4

.3048

.06309
MILLIMETERS (MM)

METERS (M)

LITERS PER SECOND (L/S) 
CULUMN 1 (WELL LOCATION) THE STANDARD METHOD OF GIVING LOCATION BY FRACTIUNAL SECTION, SECTION, TOWNSHIP, AND RANGE IS REPLACED BY THE METHOD ILLUSTRATED IN THE DIAGRAM BELOW. THE LOCATION OF THE WELL. INDICATED BY THE DDT NORMALLY WUULD BE DESCRIBED AS SE $1 / 4 \mathrm{NW} 1 / 4 \mathrm{NE} 1 / 4$ SEC.35, T. $13 \mathrm{~N} .1 \mathrm{R}$. 1 W. THE METHOD USED IN THIS REPORT REVERSES THE ORDER AND INDICATES QUARTER SUBDIVISIONS OF THE SECTION BY LETTERS. BY THIS METHOD THE LOCATION OF THE WELL IS GIVEN AS 13N-01W-35ABD 1. THE FINAL DIGIT (1) IS THE SEQUENTIAL NUMBER OF A WELL WITHIN THE SMALLEST FRACTIONAL SUBDIVISION.

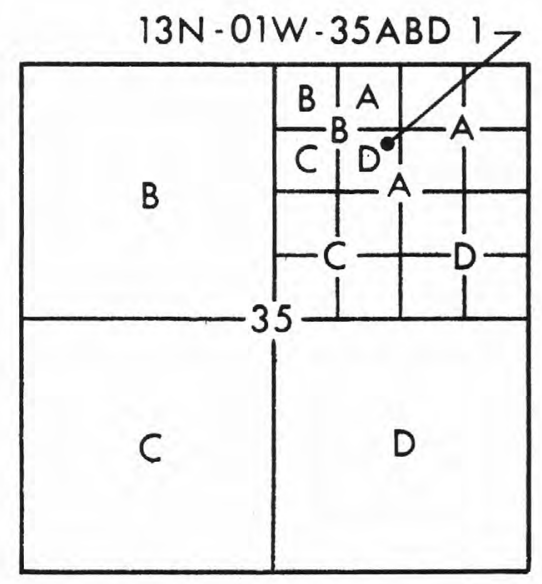

CULUMN 2 (UNNER UR USER) OWNER OR USER WHEN THE WELL WAS INVENTORIED.

COLUMN 3 (YEAR DRILLED) MAY BE ESTIMATED OR REPORTED.

COLUMN 4 (WELL DEPTH) DEPTH DF THE WELL BELOW LAND SURFACE TO THE NEAREST FODT. NUMBERS FOLLOWING THE DEPTH OF THE WELL ARE ACCURACY CODES AS FOLLOWS:

\footnotetext{
0. ACCURATE WITHIN 1 FT

1. LESS THAN 1 FT

3. FROM DRILLER'S LUG

5. ESTIMATEO

6. KEPURTED
} 
C. COMMERCIAL

D. DEWATERING

H. DOMESTIC

I. IRRIGATION

N. INDUSTRIAL

P. PUBLIC SUPPLY

R. RECREATIDN

S. STOCK SUPPLY

T. INSTITUTIONAL

U. UNUSED

COLUMN 6 (WELL DIAMETER) DIAMETER OF THE WELL TO THE NEAREST INCH.

COLUMN 7 (DEPTH TO WATER) DEPTH TO THE WATER LEVEL BELOW LAND SURFACE TO THE NEAREST FODT. "+" INDICATES WATER LEVEL ABOVE LAND SURFACE. LETTERS FOLLOWING THE WATER LEVEL ARE ACCURACY CODES AS FOLLOWS:

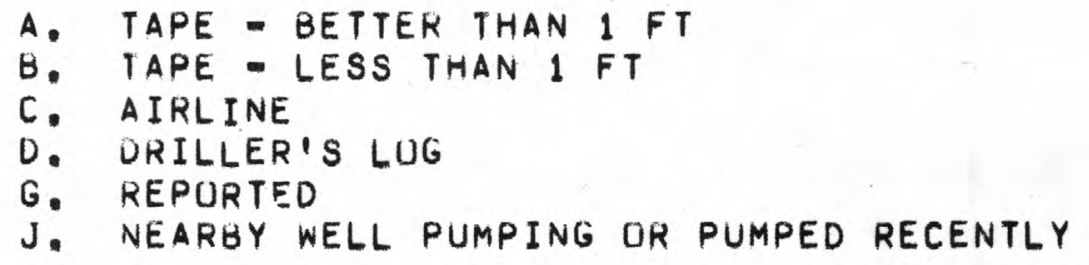

ZOLUMN 8 (DATE MEASURED) DATE OF WATER-LEVEL MEASUREMENT

:OLUMN 9 (WELL YIELD) DISCHARGE OF THE WELL IN GALLONS PER MINUTE. THE NUMBERS FOLLOWING THE WELL YIELD ARE ACCURACY CODES AS FOLLOWS:

2. PRUPELLER-TYPE METER

3. BAILER.

4. URIFICE

0 . ESTIMATED = BLANK IF REPORTED

CULUMN 10 (AQUIFERS) THE AQUIFER CODE IS:

AQUIFER OR FORMATIUN

PERMIAN

CQ GARBER-WELLINGTON FORMATIONS

CULUMN 11 (ALTITUDE OF LAND SURFACE) NUMBERS ARE IN FEET ABUVE SEA LEVEL. 
CULUMN 12 (REMARKS) THE CODE USED IS AS FOLLOWS:

D - DRILLER'S LUG

E - ELECTRIC LUG

G - GEULOGIC LOG

J - GAMMA-RAY LOG

$N$ - NEUTRON LOG

Q - WATER-QUALITY dATA AVAILABLE

R - RADIATION LOG

$Y$ - ELECTRIC, RADIATIUN, AND

DRILLER'S LOGS 
TABLE 1.--RECURDS UF WELLS IN THE GARBER-WELLINGTON AQUIFER

OKLAHOMA COUNTY

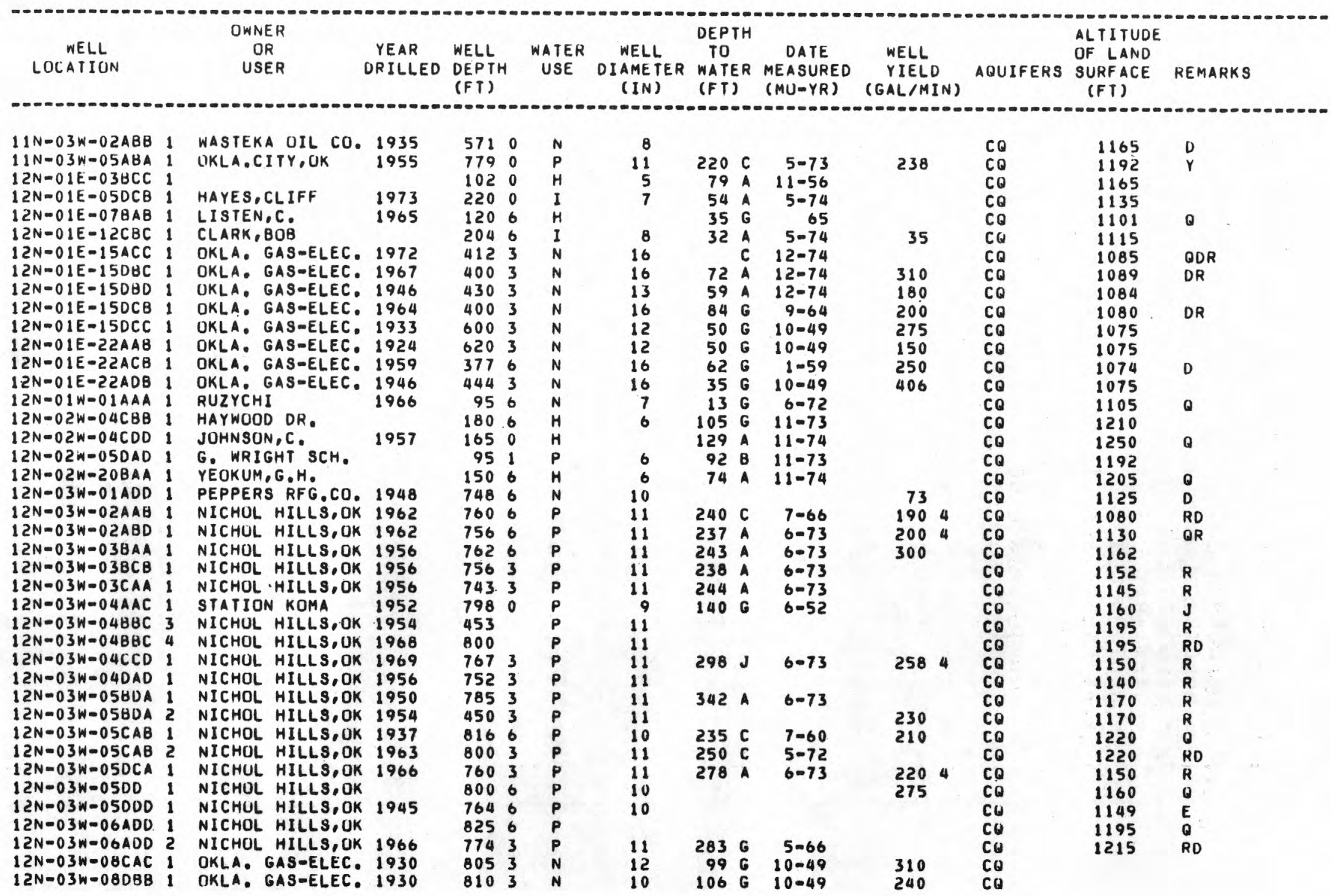




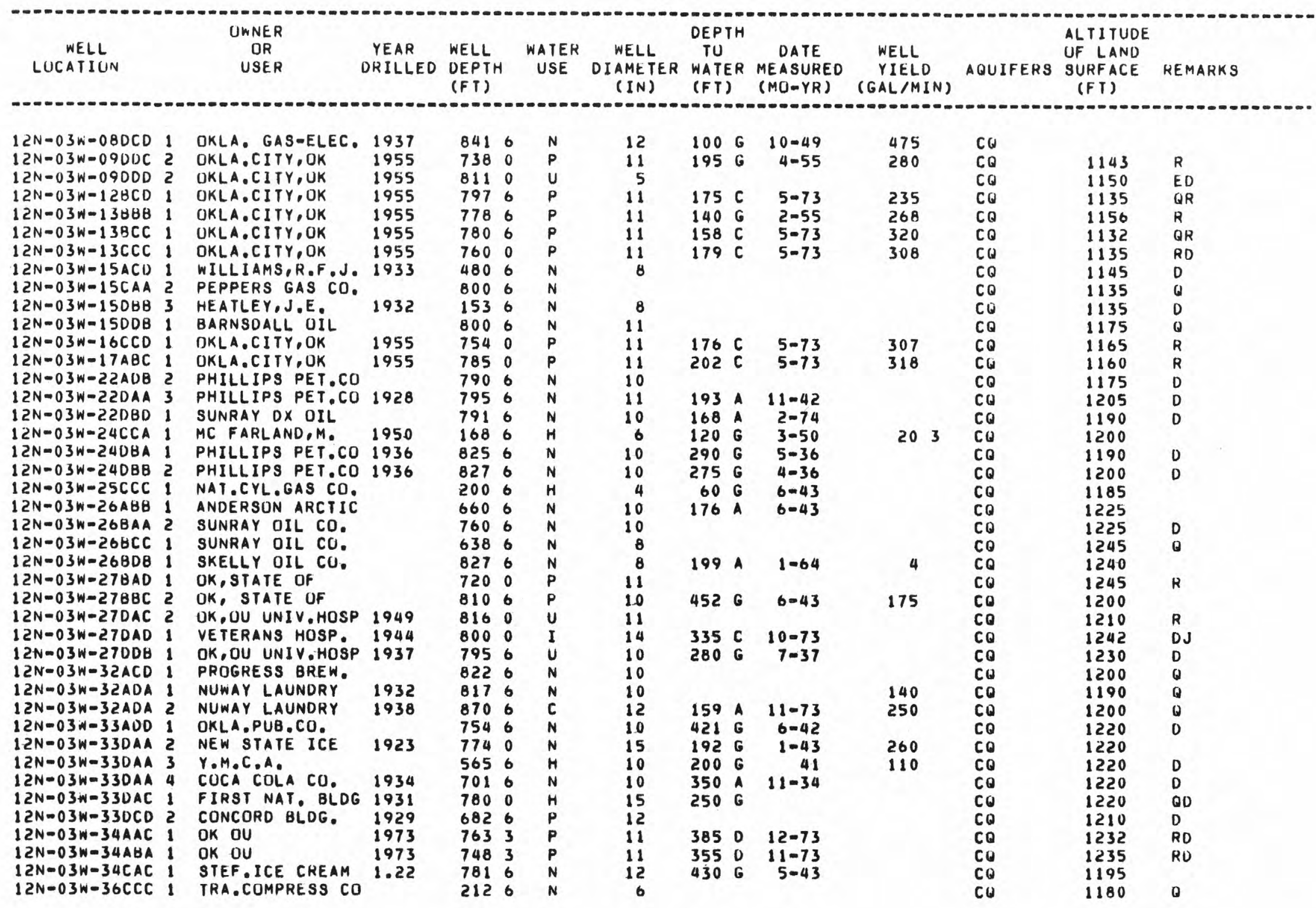




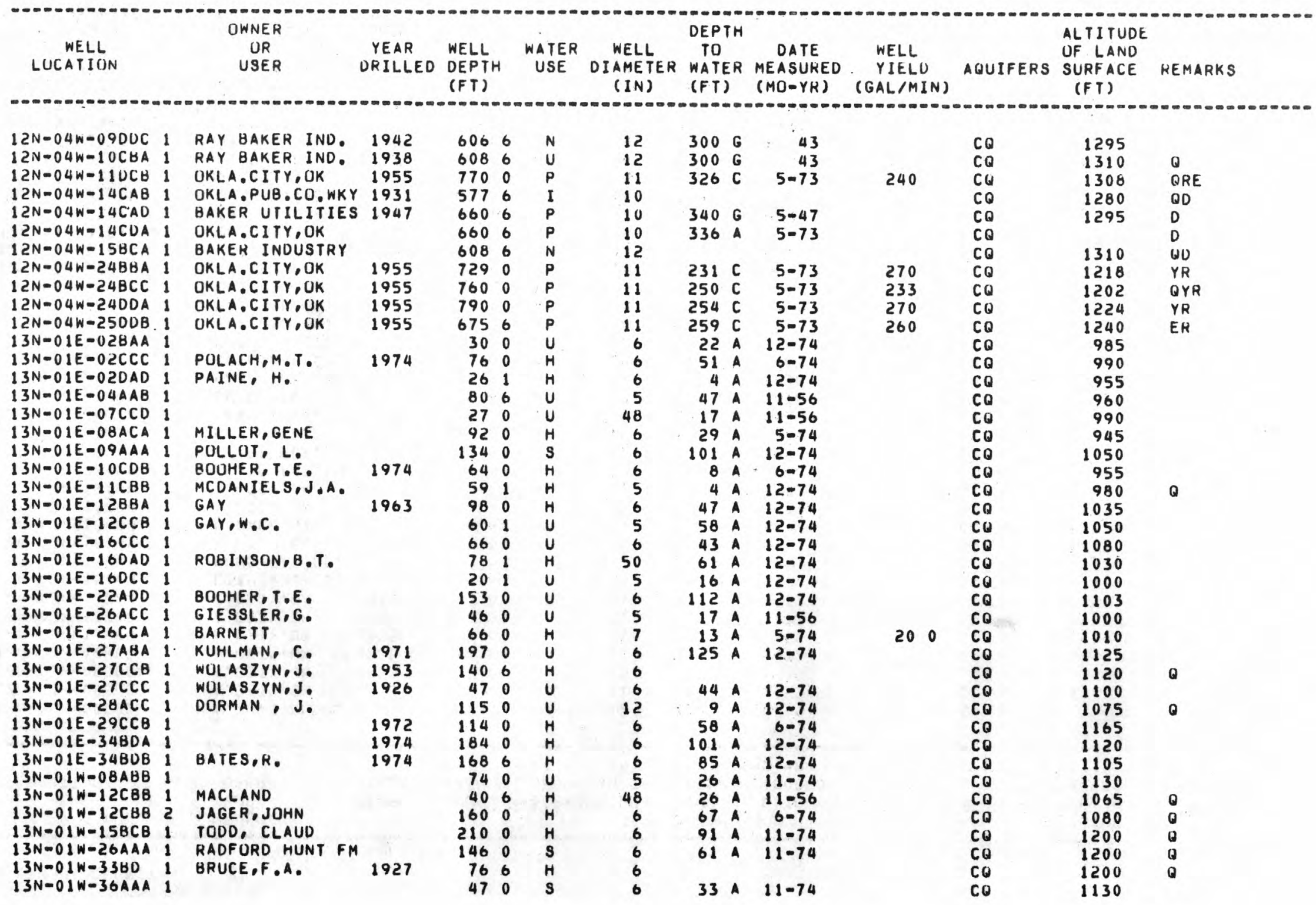




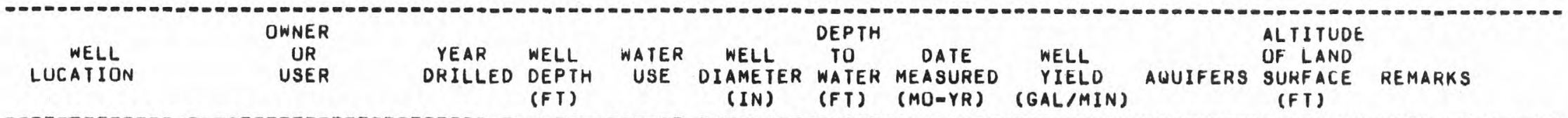

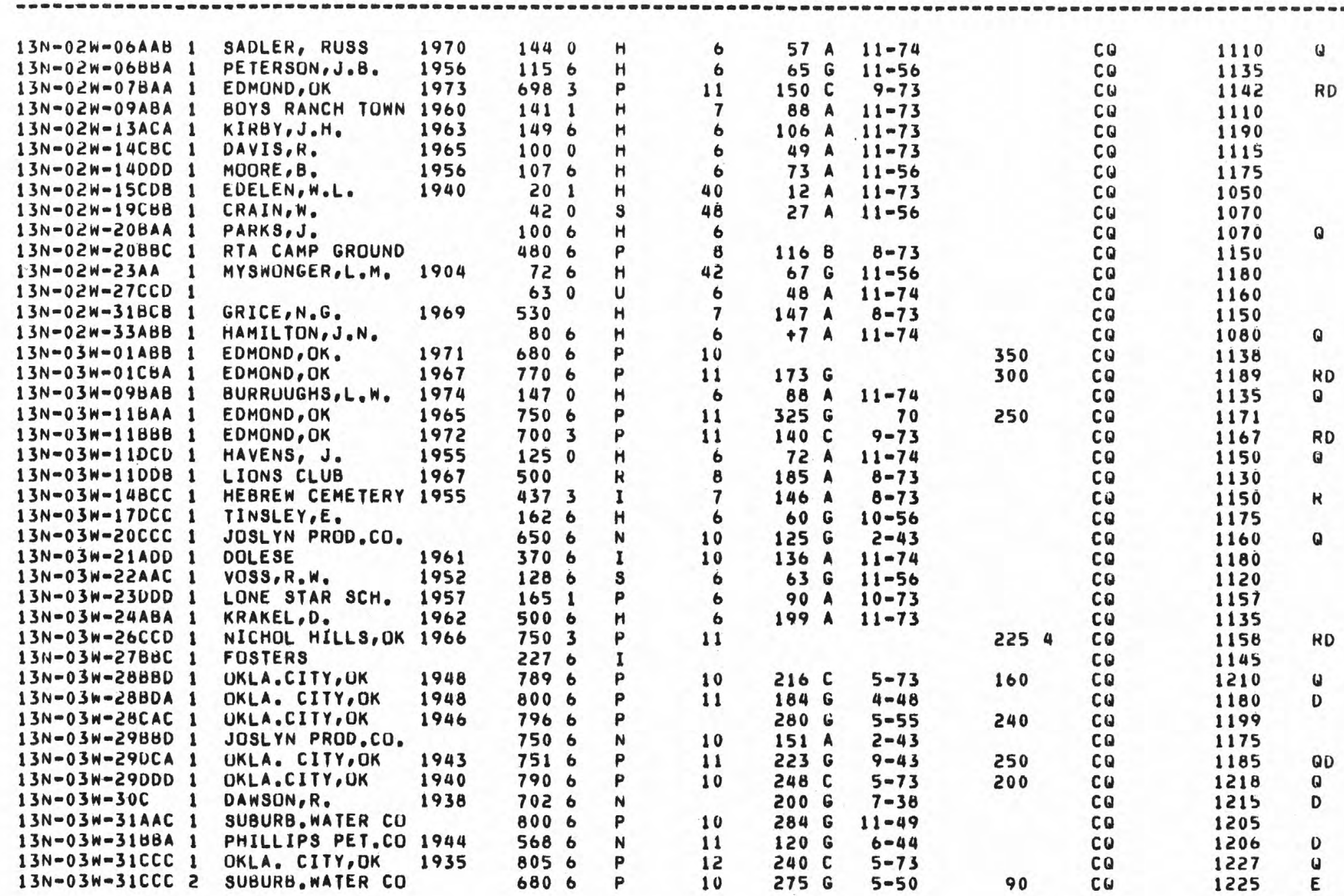




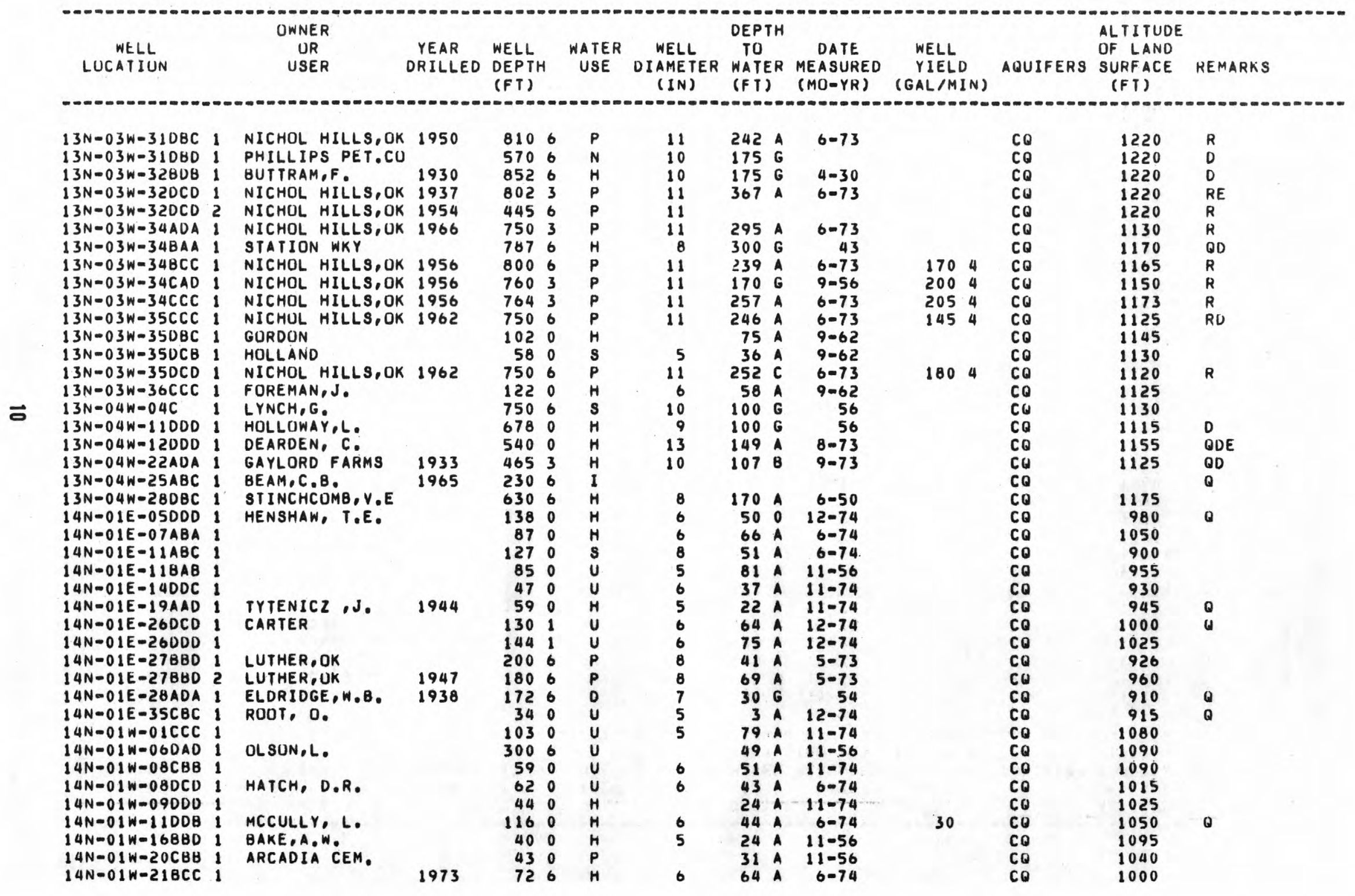




\begin{tabular}{|c|c|c|c|c|c|c|c|c|c|c|c|c|c|}
\hline $\begin{array}{l}\text { WELL } \\
\text { LUCATIUN }\end{array}$ & $\begin{array}{c}\text { OWNER } \\
\text { UR } \\
\text { USER }\end{array}$ & $\begin{array}{l}\text { YEAR } \\
\text { DRILLED }\end{array}$ & $\begin{array}{l}\text { WELL } \\
\text { DEPTH } \\
\text { (FT) }\end{array}$ & & $\begin{array}{l}\text { WATER } \\
\text { USE }\end{array}$ & $\begin{array}{l}\text { WELL } \\
\text { DIAMETER } \\
\text { (IN) }\end{array}$ & $\begin{array}{l}\text { DEPT } \\
\text { TO } \\
\text { WATE } \\
\text { (FT) }\end{array}$ & & $\begin{array}{l}\text { DATE } \\
\text { MEASURED } \\
\text { (MO-YR) }\end{array}$ & $\begin{array}{c}\text { WELL } \\
\text { YIELD } \\
\text { (GAL/MIN) }\end{array}$ & AQUIFERS & $\begin{array}{l}\text { ALTI TUDE } \\
\text { OF LANU } \\
\text { SURFACE } \\
\text { (FT) }\end{array}$ & REMARKS \\
\hline 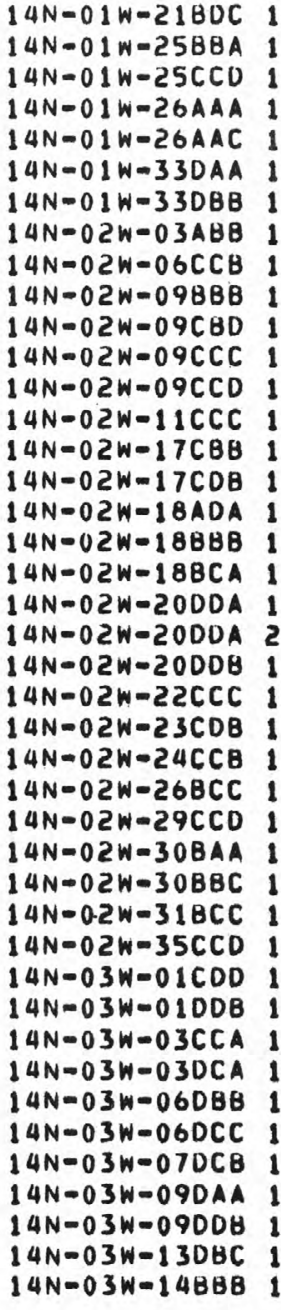 & 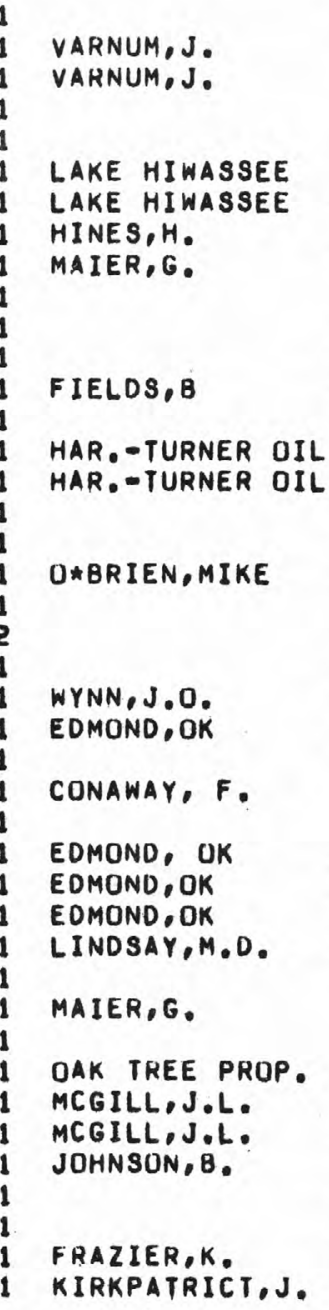 & $\begin{array}{l}1974 \\
1972 \\
1974 \\
1956 \\
1974\end{array}$ & $\begin{array}{r}16 \\
55 \\
360 \\
365 \\
102 \\
130 \\
40 \\
99 \\
122 \\
90 \\
30 \\
160 \\
635 \\
40 \\
104 \\
166 \\
46 \\
104 \\
158 \\
142 \\
420 \\
90 \\
90 \\
49 \\
749 \\
709 \\
644 \\
141 \\
77 \\
80 \\
121 \\
410 \\
650 \\
120 \\
260 \\
90 \\
128 \\
47\end{array}$ & $\begin{array}{l}0 \\
0 \\
3 \\
3 \\
0 \\
6 \\
0 \\
0 \\
0 \\
6 \\
1 \\
6 \\
6 \\
1 \\
0 \\
0 \\
0 \\
0 \\
0 \\
6 \\
3\end{array}$ & $\begin{array}{l}I \\
H \\
H \\
H \\
S \\
P \\
P \\
H \\
H \\
S \\
H \\
H \\
H \\
U \\
N \\
H \\
U \\
H \\
H \\
U \\
H \\
H \\
H \\
N \\
U \\
H \\
U \\
P \\
P \\
P \\
H \\
S \\
H \\
U \\
C \\
U \\
U \\
H \\
H \\
H \\
H \\
H\end{array}$ & $\begin{array}{r}10 \\
12 \\
8 \\
8 \\
6 \\
6 \\
8 \\
6 \\
11 \\
6 \\
6 \\
6 \\
11 \\
11 \\
6 \\
5 \\
5 \\
5 \\
15 \\
10 \\
11 \\
6 \\
8 \\
8 \\
6 \\
6\end{array}$ & 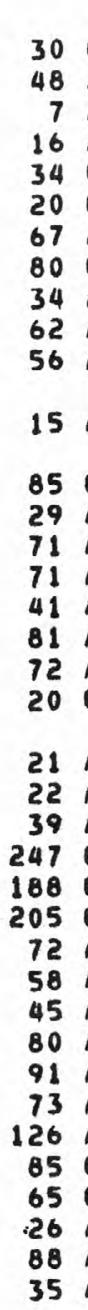 & $\begin{array}{l}G \\
A \\
A \\
A \\
G \\
G \\
A \\
G \\
A \\
A \\
A \\
A \\
G \\
A \\
A \\
A \\
A \\
A \\
A \\
G \\
A \\
A \\
A \\
G \\
G \\
C \\
A \\
A \\
A \\
A \\
A \\
A \\
A \\
G \\
G \\
A \\
A \\
A\end{array}$ & $\begin{array}{r}56 \\
11-56 \\
6-74 \\
6-74 \\
9-71 \\
9-71 \\
6-74 \\
10-73 \\
4-54 \\
6-74 \\
11-74 \\
11-73 \\
\\
43 \\
11-73 \\
12-74 \\
6-74 \\
11-56 \\
11-74 \\
7-74 \\
56 \\
11-56 \\
11-74 \\
11-74 \\
70 \\
70 \\
9-73 \\
11-74 \\
12-74 \\
11-56 \\
11-74 \\
11-74 \\
8-73 \\
8-73 \\
12-74 \\
12-74 \\
12-74 \\
11-56\end{array}$ & $\begin{array}{l}225 \\
175 \\
\\
200\end{array}$ & $\begin{array}{l}C \theta \\
C \theta \\
C \theta \\
C \theta \\
C \theta \\
C \theta \\
C \theta \\
C \theta \\
C \theta \\
C \theta \\
C \theta \\
C \theta \\
C \theta \\
C \theta \\
C \theta \\
C \theta \\
C \theta \\
C \theta \\
C \theta \\
C \theta \\
C \theta \\
C \theta \\
C \theta \\
C \theta \\
C \theta \\
C \theta \\
C \theta \\
C \theta \\
C \theta \\
C \theta \\
C \theta \\
C \theta \\
C \theta \\
C \theta\end{array}$ & $\begin{array}{r}955 \\
985 \\
9970 \\
950 \\
950 \\
980 \\
980 \\
1138 \\
1200 \\
1090 \\
1092 \\
1105 \\
1100 \\
1070 \\
1110 \\
1120 \\
1080 \\
1055 \\
1150 \\
1135 \\
1150 \\
1140 \\
1070 \\
1010 \\
1030 \\
1053 \\
1100 \\
1210 \\
1172 \\
1095 \\
1060 \\
1178 \\
1140 \\
1145 \\
1136 \\
1075 \\
1100 \\
1095 \\
1060 \\
1050 \\
1065 \\
1117\end{array}$ & $\begin{array}{l}\text { QR } \\
0 \\
0 \\
D \\
R D \\
0\end{array}$ \\
\hline
\end{tabular}


OKLAHUMA CUUNTY

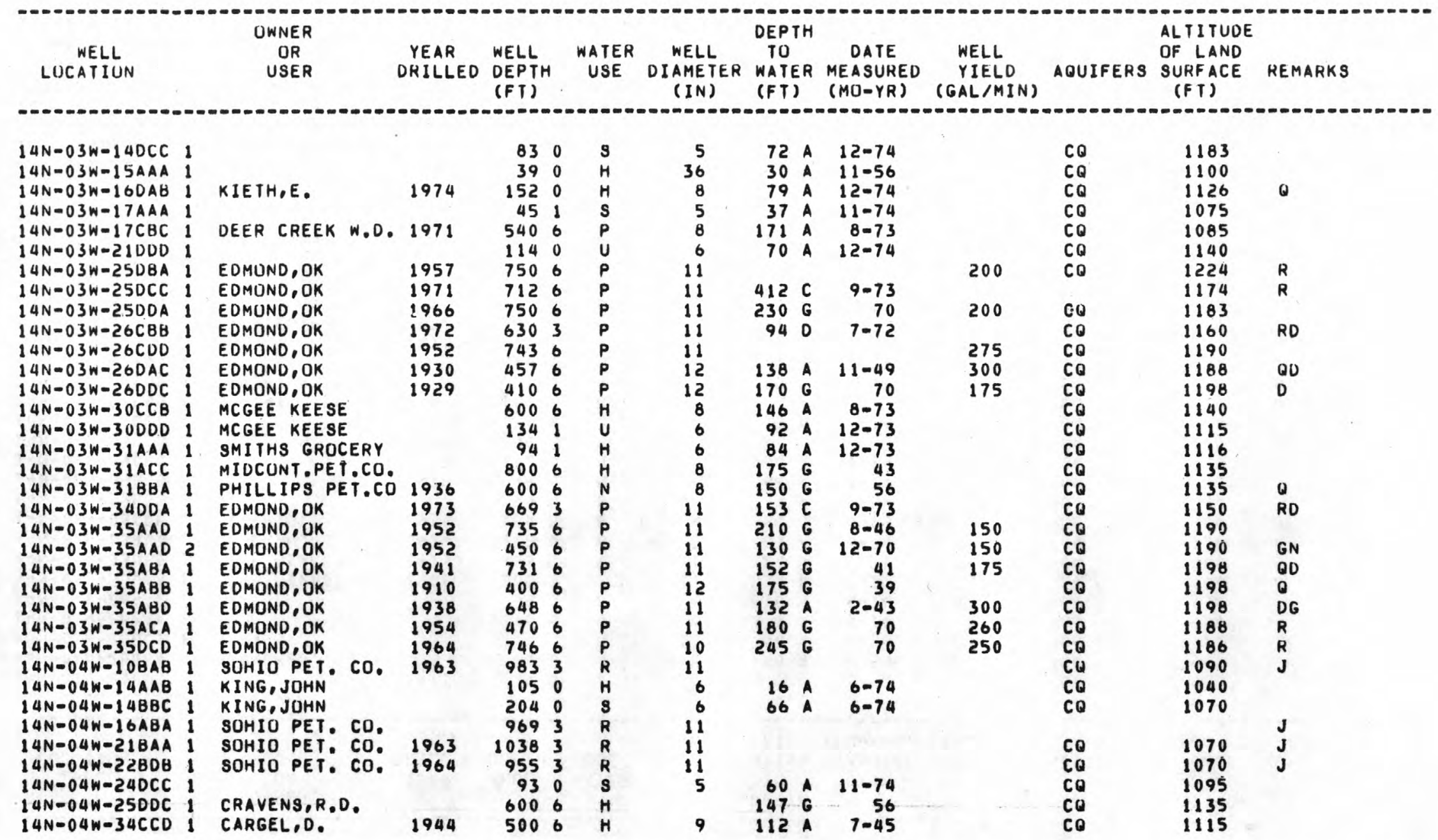




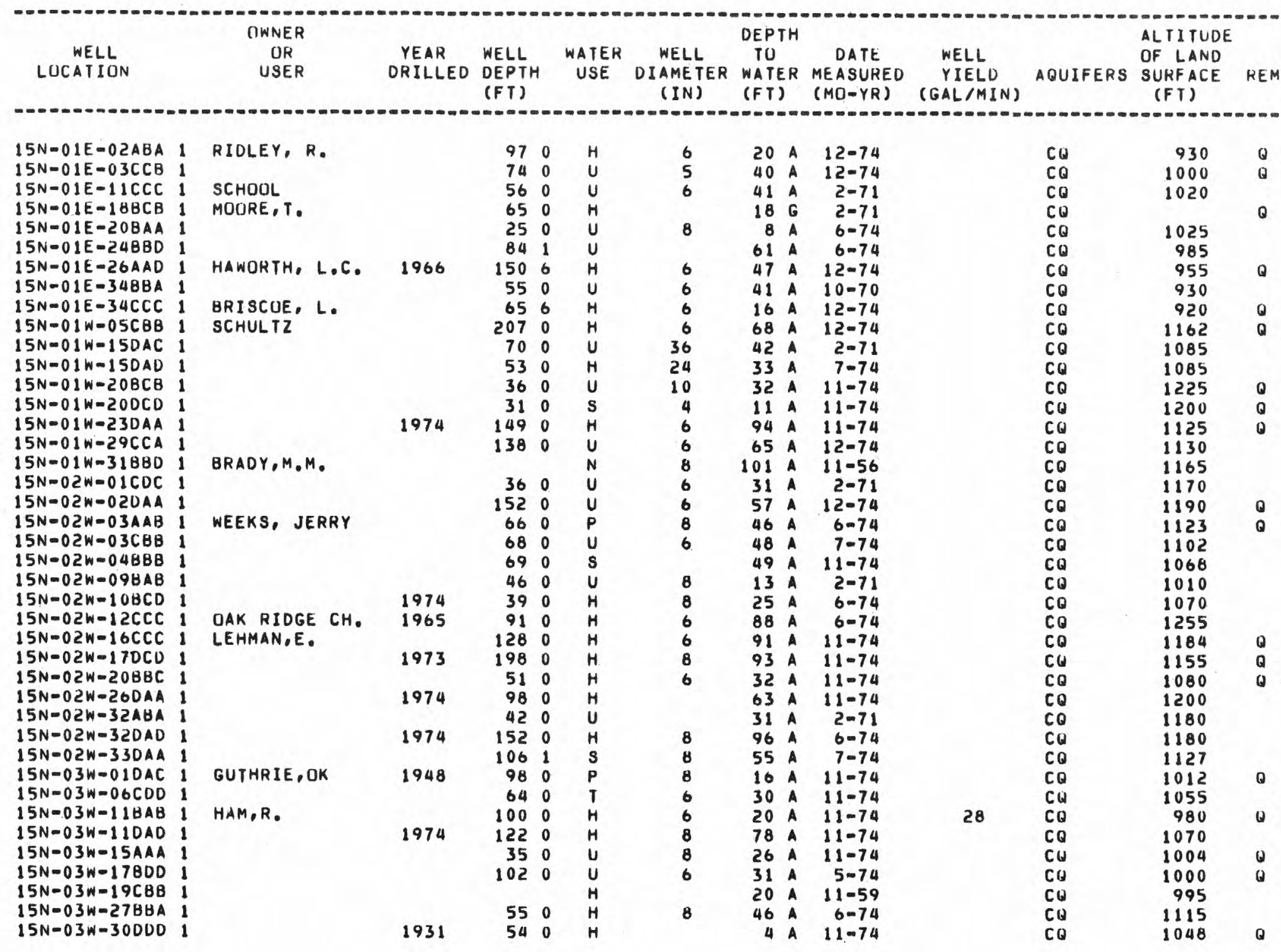




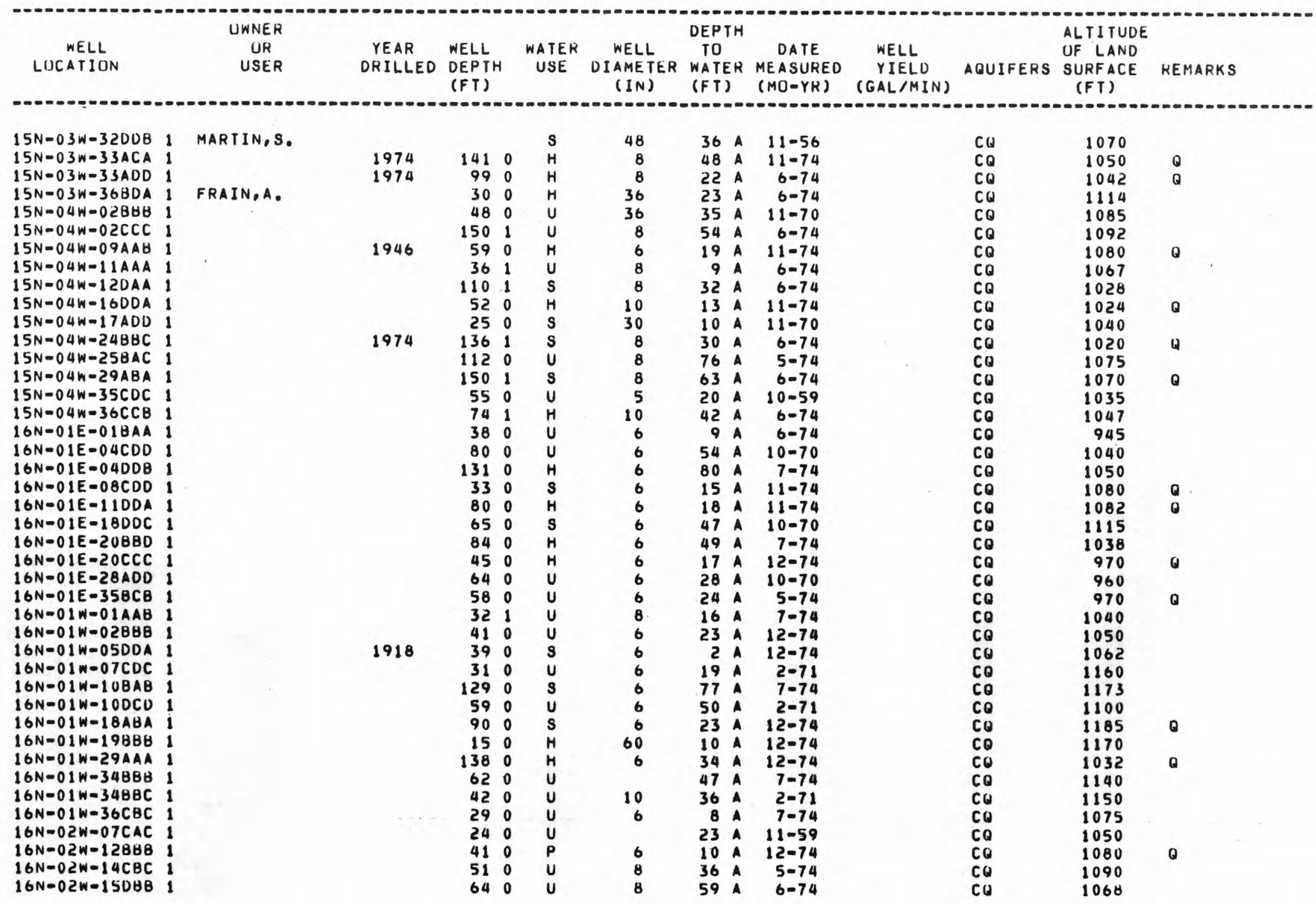




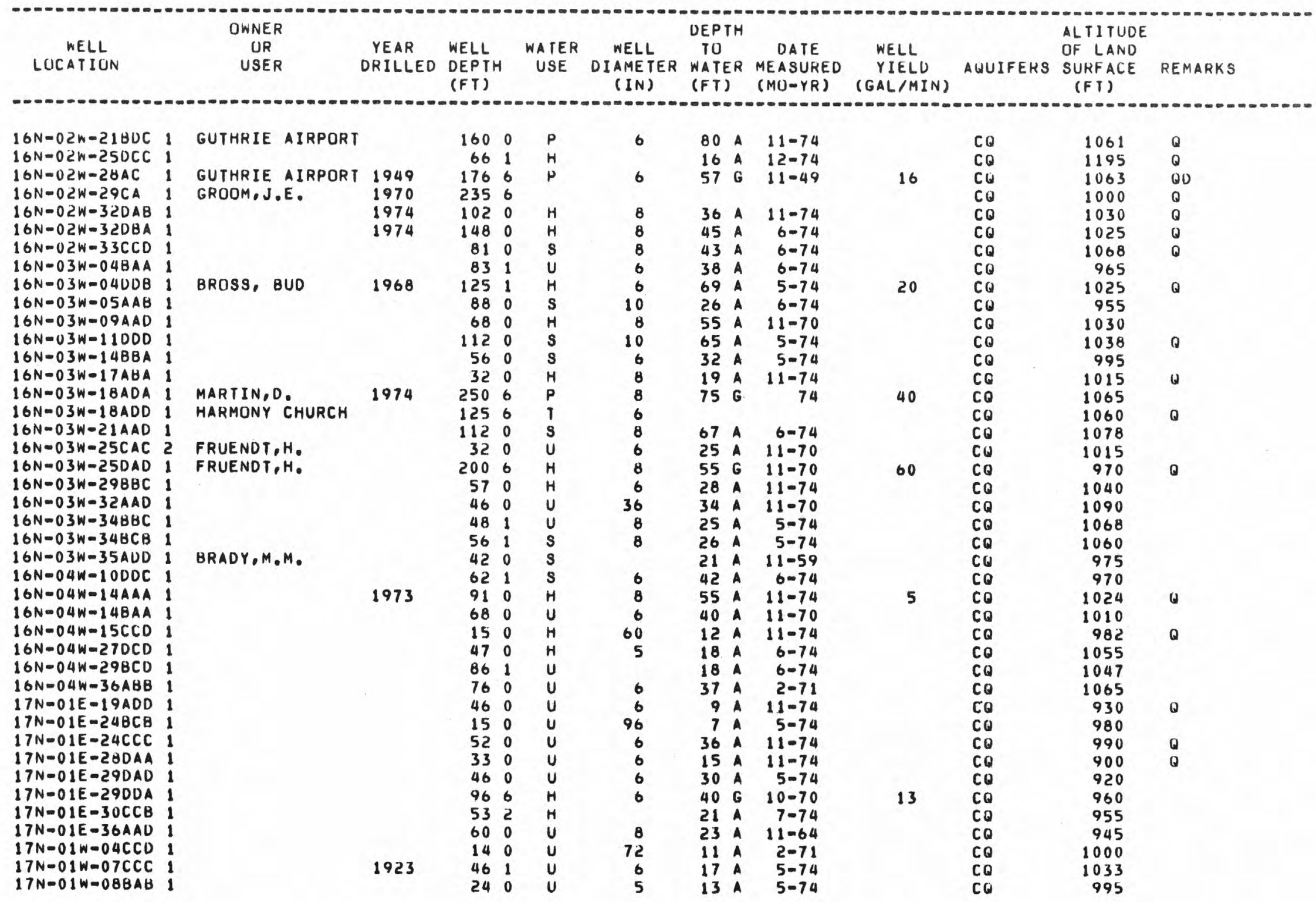


LUGAN CUUNTY

\begin{tabular}{|c|c|c|c|c|c|c|c|c|c|c|c|c|}
\hline $\begin{array}{c}\text { WELL } \\
\text { LUCATION }\end{array}$ & $\begin{array}{c}\text { OWNER } \\
\text { OR } \\
\text { USER }\end{array}$ & $\begin{array}{c}\text { YEAR } \\
\text { DRILLED }\end{array}$ & $\begin{array}{l}\text { WELL } \\
\text { DEPTH } \\
\text { (FT) }\end{array}$ & $\begin{array}{l}\text { WATER } \\
\text { USE }\end{array}$ & $\begin{array}{l}\text { WELL } \\
\text { DIAMETER } \\
\text { (IN) }\end{array}$ & $\begin{array}{l}\text { DEPT } \\
\text { TO } \\
\text { WATE } \\
\text { (FT) }\end{array}$ & & $\begin{array}{l}\text { DATE } \\
\text { MEASURED } \\
\text { (MO-YR) }\end{array}$ & $\begin{array}{c}\text { WELL } \\
\text { YIELD } \\
\text { (GAL/MIN) }\end{array}$ & AQUIFERS & $\begin{array}{l}\text { ALTITUDE } \\
\text { UF LAND } \\
\text { SURFACE } \\
\text { (FT) }\end{array}$ & REMARKS \\
\hline $\begin{array}{l}17 N-01 W-21 C B A \\
17 N-01 W-21 C B B \\
17 N=01 W-26 B C B \\
17 N-01 W-32 A B B \\
11 N-01 W-32 B A B \\
17 N-01 W-35 B B B \\
17 N-02 W=02 D C D \\
17 N-02 W-14 B C B \\
17 N-02 W-15 D 0 D \\
17 N-02 W-22 C D B \\
17 N=02 W=34 A A A \\
17 N-02 W-34 D A A\end{array}$ & $\begin{array}{ll}1 & \\
1 & \text { BROWN } \\
1 & \\
1 & \\
1 & \\
1 & \\
1 & \\
1 & \\
1 & \\
1 & \\
1 & \\
1 & \end{array}$ & 1974 & $\begin{array}{r}92 \\
186 \\
24 \\
51 \\
49 \\
45 \\
23 \\
30 \\
48 \\
78 \\
200 \\
190\end{array}$ & $\begin{array}{l}H \\
H \\
U \\
H \\
H \\
U \\
U \\
U \\
U \\
U \\
H \\
H\end{array}$ & $\begin{array}{r}6 \\
6 \\
8 \\
6 \\
36 \\
6 \\
6 \\
6 \\
8 \\
6 \\
6\end{array}$ & $\begin{array}{r}58 \\
108 \\
19 \\
46 \\
44 \\
4 \\
20 \\
24 \\
44 \\
39 \\
67 \\
27\end{array}$ & $\begin{array}{l}\mathbf{A} \\
\mathbf{A} \\
\mathbf{A} \\
\mathbf{A} \\
\mathbf{A} \\
\mathbf{A} \\
\mathbf{A} \\
\mathbf{A} \\
\mathbf{A} \\
\mathbf{A} \\
\mathbf{A} \\
\mathbf{A}\end{array}$ & $\begin{array}{r}7=74 \\
11=74 \\
2=71 \\
7=74 \\
2=71 \\
12=74 \\
2=71 \\
2=71 \\
5=75 \\
5=74 \\
11=74 \\
11=74\end{array}$ & & $\begin{array}{l}C Q \\
C Q \\
C Q \\
C Q \\
C Q \\
C Q \\
C Q \\
C Q \\
C Q \\
C Q \\
C Q \\
C Q\end{array}$ & $\begin{array}{r}1075 \\
1082 \\
970 \\
1082 \\
1110 \\
1032 \\
990 \\
970 \\
970 \\
990 \\
1018 \\
980\end{array}$ & $\begin{array}{l}0 \\
0 \\
0\end{array}$ \\
\hline
\end{tabular}

क 
EXPLANATION OF INFORMATION IN TABLE 2

WELL-NUMUERING SYSTEM IS GIVEN IN EXPLANATION FOR TABLE 1. date - date sample collected

EXPLANATION OF ABBREVIATIONS:

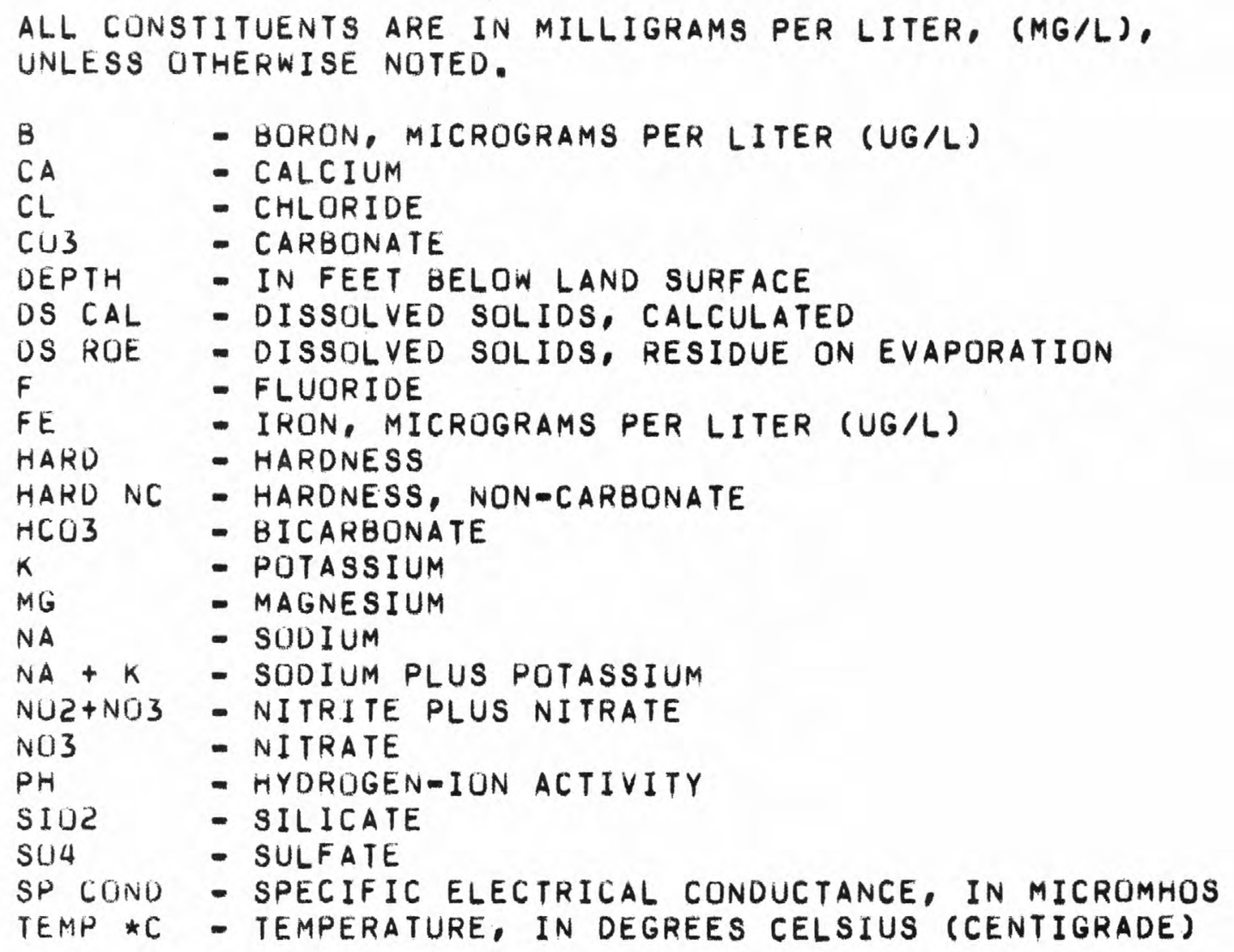


TABLE 2.--CHEMICAL ANALYSES UF GRUUNO WATER FRUM THE GARGER-WELLINGTON AQUIFER

OKLAHUMA COUNTY

\begin{tabular}{|c|c|c|c|c|c|c|c|c|c|c|c|}
\hline $\begin{array}{r}12 N=01 E=07 B A B \\
\text { OATE }\end{array}$ & $1(M-D=$ & $\begin{array}{l}\text { AT }=35-2 \\
): 08-27-7\end{array}$ & $\begin{array}{l}9-38 \\
2\end{array}$ & LONG. - 09 & $7-14-29$ & $\operatorname{SEQ} U_{0}-1$ & & & & & \\
\hline $\begin{array}{l}\text { CA } \cdots \cdots \\
\text { NO3 } \cdots \\
\text { TEMP } \Vdash C\end{array}$ & $\begin{array}{r}70.0 \\
.4 \\
25.0\end{array}$ & 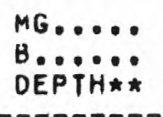 & $\begin{array}{r}26.0 \\
120.1 \\
\end{array}$ & $\begin{array}{l}\text { NA. } . . . . \\
\text { DS CAL. }\end{array}$ & $\begin{array}{l}180.0 \\
780.0\end{array}$ & $\begin{array}{l}\text { HCU3... } \\
\text { HARU... }\end{array}$ & $\begin{array}{l}340.0 \\
280.0\end{array}$ & SO4 COAB & $\begin{array}{r}76.0 \\
1330.0\end{array}$ & $\begin{array}{l}C L \ldots \ldots \\
P H \ldots \ldots\end{array}$ & $\begin{array}{r}220.0 \\
8.2\end{array}$ \\
\hline $\begin{array}{r}12 N-01 E-15 A C C \\
\text { DATE }\end{array}$ & $1(M-D=$ & AT: $=\begin{array}{r}35=3 \\
: 12-18=7\end{array}$ & $0-52$ & LONG. $=09$ & $7-11-07$ & $S E Q_{0}-1$ & & & & & \\
\hline $\begin{array}{l}\text { DEPTH* } \\
\text { SO4.... } \\
\text { PH.... }\end{array}$ & $\begin{array}{r}412.0 \\
50.0 \\
7.8\end{array}$ & $\begin{array}{l}C A \ldots \ldots \\
C L \ldots \ldots \\
M G \ldots \ldots\end{array}$ & $\begin{array}{l}52.0 \\
80.0 \\
31.0\end{array}$ & $\begin{array}{l}\text { NA. } \\
\text { OS ROE. }\end{array}$ & $\begin{array}{r}76.0 \\
473.0\end{array}$ & K....... & $\begin{array}{r}3.6 \\
260.0\end{array}$ & $\begin{array}{l}\text { HCO3 } \cdots: ~ \\
\text { HARD NC }\end{array}$ & 335.0 & SP COND & 836.0 \\
\hline $\begin{array}{r}12 N=0 I W=01 \text { AAA } \\
\text { DATE }\end{array}$ & $E^{1}(M-D-$ & $\begin{array}{l}A T=-35-3 \\
\text { S: } 06-13=7\end{array}$ & $3-02$ & LONG. $=09$ & $7-14-52$ & $S E Q_{0}-1$ & & & & & \\
\hline $\begin{array}{l}\text { NA..... } \\
\text { DS ROE } \\
\text { DEPTHћћ }\end{array}$ & $\begin{array}{r}63.0 \\
430.0 \\
95.0\end{array}$ & $\begin{array}{l}\mathrm{HCO} 3 \ldots \\
\mathrm{CO} 3 \ldots\end{array}$ & 434.0 & $\begin{array}{l}\text { SO4.... } \\
\text { HARD... }\end{array}$ & $\begin{array}{r}35.0 \\
280.0\end{array}$ & SLP COND & $\begin{array}{r}16.0 \\
733.0\end{array}$ & $\begin{array}{l}\mathrm{NO3} \ldots \ldots \\
\mathrm{PH} \ldots \ldots\end{array}$ & 7.18 & TEMP $\star \dot{C}$ & 19.1 \\
\hline $\begin{array}{r}12 N=02 W=04 C D D \\
\text { DATE }\end{array}$ & $E^{\prime}(M-D=$ & $\begin{array}{l}\text { AT: }=35=3 \\
\text { ) }: 11-11=7\end{array}$ & $\begin{array}{l}2-15 \\
4\end{array}$ & LONG. $=09$ & $7-24-56$ & $\operatorname{SEQ},-1$ & & & & & \\
\hline 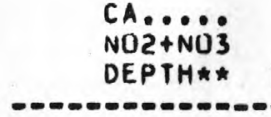 & $\begin{array}{r}61.0 \\
6.3 \\
165.0\end{array}$ & $\begin{array}{l}\text { MG.PB̈: } \\
\text { DS ROE. }\end{array}$ & $\begin{array}{r}29.0 \\
342.0\end{array}$ & $\begin{array}{l}\text { NA..... } \\
\text { HARDE... }\end{array}$ & $\begin{array}{r}26.0 \\
270.0\end{array}$ & $\begin{array}{l}\text { HCU3 } . \ddot{~} \\
\text { HARO NC }\end{array}$ & $\begin{array}{r}330.0 \\
1.0\end{array}$ & SU4 COND & $\begin{array}{r}12.0 \\
590.0\end{array}$ & $\begin{array}{l}C L \ldots \ldots \\
P H \ldots \ldots\end{array}$ & $\begin{array}{r}15.0 \\
7.8\end{array}$ \\
\hline $\begin{array}{r}12 N-02 W=208 A A \\
\text { DATE }\end{array}$ & $E^{\prime}(M-D-$ & 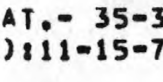 & $\begin{array}{l}0-21 \\
4\end{array}$ & LONG $=0$ & $7-25-59$ & $S E Q_{0}-1$ & & & & & \\
\hline${ }_{\mathrm{NO}}^{\mathrm{CA}+\mathrm{NU}}$ & $\begin{array}{r}58.0 \\
4.1\end{array}$ & $\begin{array}{l}\text { MG } \\
\text { DS ROË. }\end{array}$ & $\begin{array}{r}23.0 \\
348.0\end{array}$ & $\begin{array}{l}\text { NA..... } \\
\text { HARD } \ldots\end{array}$ & $\begin{array}{r}45.0 \\
240.0\end{array}$ & $\begin{array}{l}\text { HCU3 ... } \\
\text { HARO NC }\end{array}$ & 343.0 & $\begin{array}{l}\text { SOL } \\
\text { SP COND }\end{array}$ & $\begin{array}{r}11.0 \\
596.0\end{array}$ & 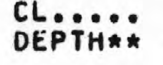 & $\begin{array}{r}15.0 \\
150.0\end{array}$ \\
\hline
\end{tabular}


OKLAHOMA COUNTY

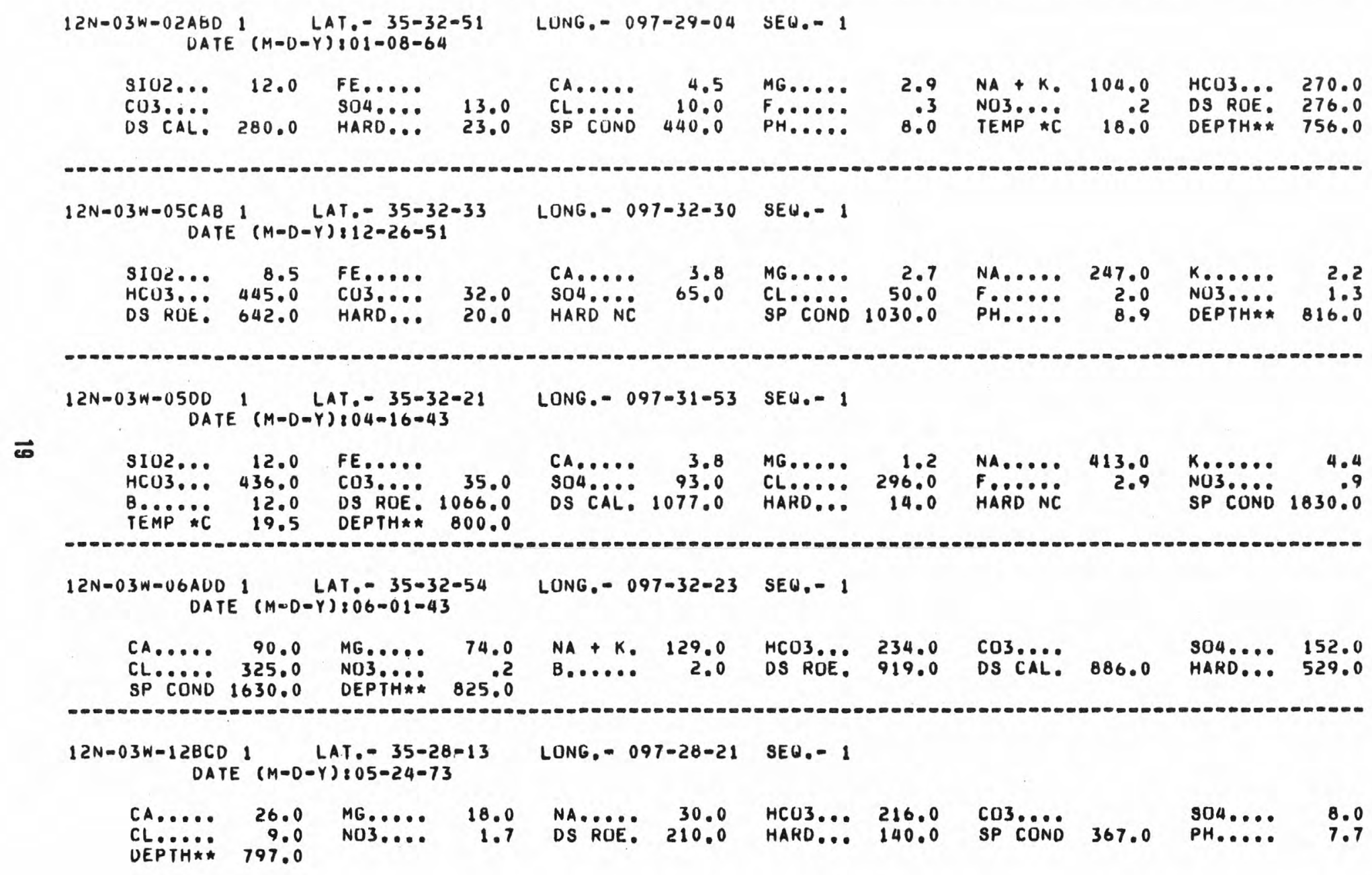




\section{OKLAHUMA CUUNTY}

\begin{tabular}{|c|c|c|c|c|c|c|c|c|c|c|c|}
\hline $\begin{array}{r}12 N=03 W-13 B C C \\
\text { DATE }\end{array}$ & $1(M-D=$ & $\begin{array}{l}A T=35-3 \\
\text { S: } 05-24-7\end{array}$ & -54 & LONG.- 09 & $7-31-26$ & $\operatorname{SEQ}=-1$ & & & & & \\
\hline $\begin{array}{l}C A \cdots \cdots \\
C L \cdots \cdots \\
\text { DEPTH* }\end{array}$ & $\begin{array}{r}31.0 \\
10.0 \\
780.0\end{array}$ & $\begin{array}{l}\text { MG } \ldots \ldots \\
\text { NO3.... }\end{array}$ & $\begin{array}{r}23.0 \\
2.0\end{array}$ & $\begin{array}{l}\text { NA. } \\
\text { DS ROE. }\end{array}$ & $\begin{array}{r}15.0 \\
208.0\end{array}$ & $\begin{array}{l}\text { HCU3 } \ldots \\
\text { HARD... }\end{array}$ & $\begin{array}{l}220.0 \\
170.0\end{array}$ & SU3 COND & 371.0 & $\begin{array}{l}\mathrm{SO} \ldots \ldots \\
\mathrm{PH} \ldots \ldots\end{array}$ & $\begin{array}{l}9.0 \\
7.8\end{array}$ \\
\hline $\begin{array}{r}12 N-03 W=150 D B \\
\text { DATE }\end{array}$ & $1(M-D=$ & $\begin{array}{l}\text { AT: }=35-3 \\
\text { ) }: 04-17=4\end{array}$ & -40 & LONG. -09 & $7-29-55$ & $S E Q_{0}-1$ & & & & & \\
\hline $\begin{array}{l}\text { CA... } \\
\text { CL } \ldots \cdots \\
\text { HARD NC }\end{array}$ & $\begin{array}{l}70.0 \\
33.0\end{array}$ & $\begin{array}{l}\text { MG..... } \\
\text { F. } \\
\text { SP CUND }\end{array}$ & $\begin{array}{r}37.0 \\
749.0\end{array}$ & $\begin{array}{l}\text { NA }+K . \\
\text { NO3. } \\
\text { DEPTH** }\end{array}$ & $\begin{array}{r}40.0 \\
10.0 \\
800.0\end{array}$ & $\begin{array}{l}\mathrm{HCO} \ldots \ldots \\
\forall \ldots \ldots\end{array}$ & $\begin{array}{r}417.0 \\
.3\end{array}$ & $\begin{array}{l}\text { CO3 } \\
\text { DS CAL. }\end{array}$ & 485.0 & $\begin{array}{l}\text { SOL } \ldots . . . \\
\text { HARD } \ldots\end{array}$ & $\begin{array}{r}17.0 \\
326.0\end{array}$ \\
\hline $\begin{array}{r}12 N-03 W-26 B C C \\
\text { DATE }\end{array}$ & $E^{\prime}(M-0=$ & $\begin{array}{l}\text { AT }=35=2 \\
: 04-17=4\end{array}$ & -15 & LONG,-09 & $7-29-30$ & $\operatorname{SEQ} \cdot-1$ & & & & & \\
\hline $\begin{array}{l}F E \cdots \cdots \\
C L \cdots \bullet \bullet\end{array}$ & 49.0 & CA, & $\begin{array}{r}37.0 \\
2.5\end{array}$ & 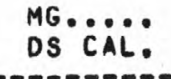 & $\begin{array}{r}31.0 \\
300.0\end{array}$ & $\begin{array}{l}\text { NA + K. } \\
\text { HARD... }\end{array}$ & $\begin{array}{r}37.0 \\
220.0\end{array}$ & $\begin{array}{l}\text { HCO3 } \\
\text { SP COND }\end{array}$ & $\begin{array}{l}264.0 \\
573.0\end{array}$ & $\begin{array}{l}\text { SO4 } \\
\text { DEPTH* }\end{array}$ & $\begin{array}{r}13.0 \\
638.0\end{array}$ \\
\hline $\begin{array}{r}12 N-03 W-32 A C D \\
\text { DATE }\end{array}$ & $E^{1}(M-D-$ & $\begin{array}{l}\text { AT }=-35-2 \\
\text { : } 04-13=4\end{array}$ & -18 & LONG $=09$ & $7-32=00$ & $S E U_{0}=1$ & & & & & \\
\hline 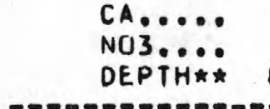 & $\begin{array}{r}53.0 \\
822.6\end{array}$ & $\begin{array}{l}M G \ldots \ldots \\
B \ldots \ldots\end{array}$ & $\begin{array}{r}25.0 \\
2.7\end{array}$ & $\begin{array}{l}\text { NA }+K . \\
\text { OS ROE. }\end{array}$ & $\begin{array}{l}137.0 \\
550.0\end{array}$ & $\begin{array}{l}\text { HCO3... } \\
\text { DS.CAL. }\end{array}$ & $\begin{array}{l}441.0 \\
574.0\end{array}$ & $\begin{array}{l}\mathrm{CO} \ldots \ldots \\
\mathrm{CL} \ldots \ldots\end{array}$ & 67.0 & $\begin{array}{l}\text { SO4 } \\
\text { SP COND }\end{array}$ & $\begin{array}{r}74.0 \\
978.0\end{array}$ \\
\hline $\begin{array}{r}12 N=03 W-32 A D A \\
\text { DATE }\end{array}$ & $1(M-D=$ & $\begin{array}{l}A T:=35-2 \\
): 04=14-4\end{array}$ & -54 & LONG. $=09$ & $7-32-06$ & $S E Q_{0}-1$ & & & & & \\
\hline $\begin{array}{l}\text { NA }+K \\
\text { NO3. } \\
\text { DEPTH** }\end{array}$ & $\begin{array}{r}187.0 \\
1.6 \\
817.0\end{array}$ & $\begin{array}{l}\mathrm{HCO} 3 \ldots \\
\mathrm{B} \ldots \ldots\end{array}$ & $\begin{array}{r}364.0 \\
10.0\end{array}$ & $\begin{array}{l}\text { CO3.... } \\
\text { DS ROE. }\end{array}$ & $\begin{array}{r}35.0 \\
420.0\end{array}$ & $\begin{array}{l}\text { SO4..... } \\
\text { HARD... }\end{array}$ & $\begin{array}{r}22.0 \\
6.0\end{array}$ & 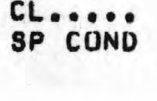 & $\begin{array}{r}21.0 \\
746.0\end{array}$ & TEMP $\bullet \dot{C}$ & 18.0 \\
\hline
\end{tabular}




\section{OKLAHOMA COUNTY}

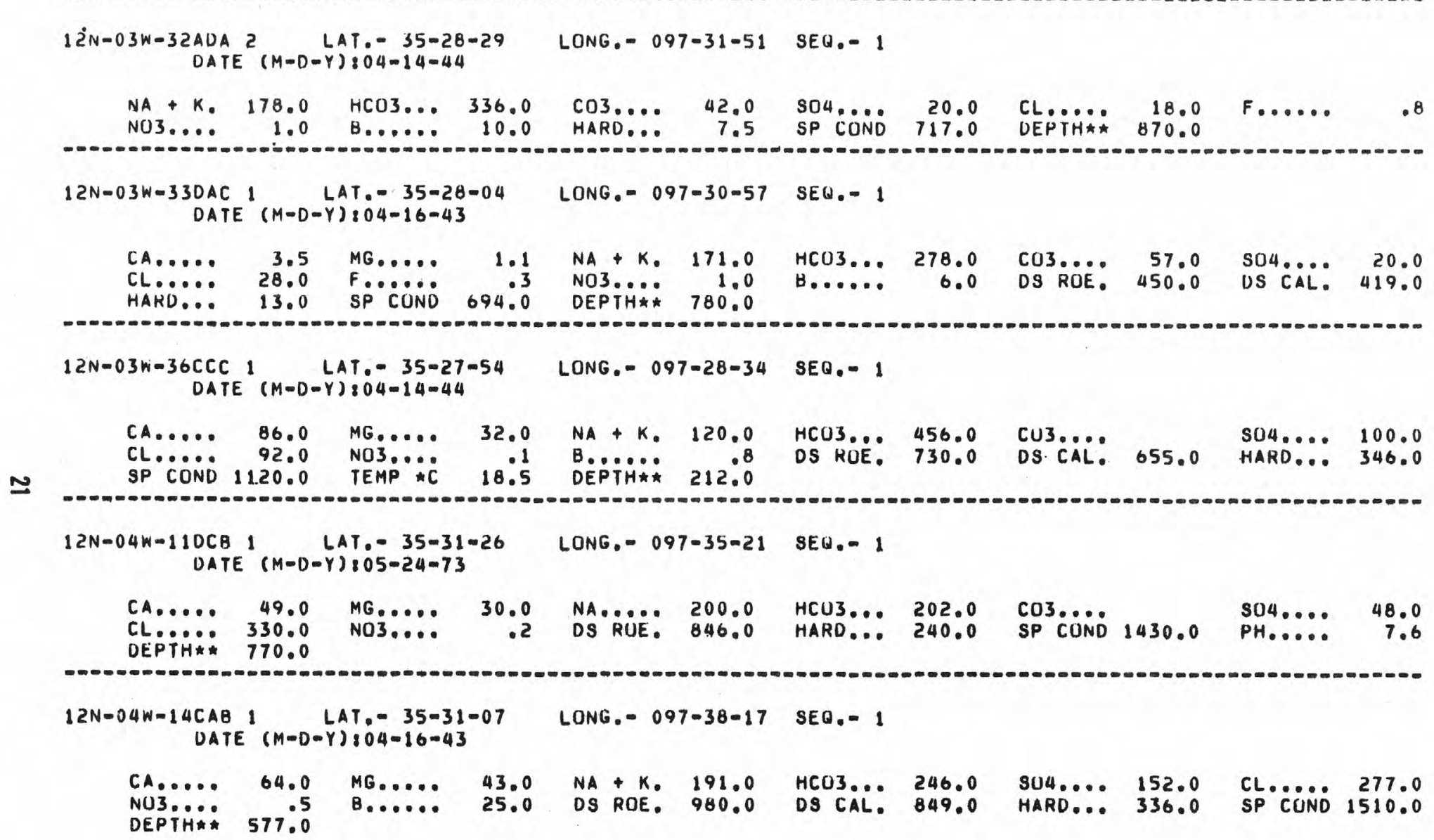


OKLAHUMA CIJUNTY

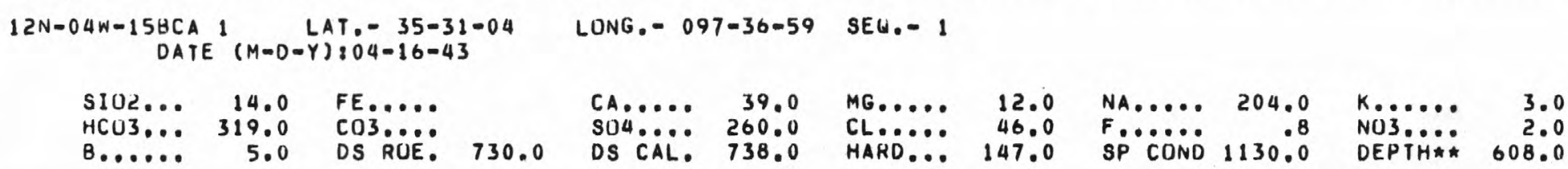

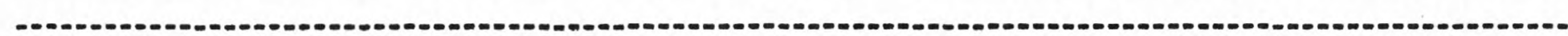

$12 N=04 W-24 B C C 1 \quad$ LAT $-35-30-26 \quad$ LONG.- 097-34-50 SEQ.- 1

DATE $(M-D-Y): 05-22-73$

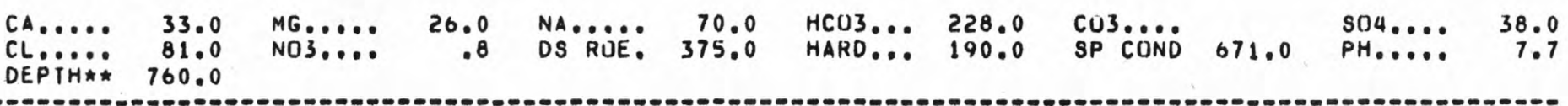

$13 N=01 E-27$ CCB $1 \quad$ LAT- $35-34-07$ LONG.- 097-11-33 SEQ.- 1

DATE $(M-D-Y): 10=22-54$

N

\begin{tabular}{|c|c|c|c|c|c|c|c|c|c|c|c|}
\hline $\begin{array}{l}\text { SIUZ... } \\
\text { HCOJ .. } \\
\text { DS RUE. } \\
\text { TEMP *C }\end{array}$ & $\begin{array}{r}14.0 \\
387.0 \\
326.0 \\
18.5\end{array}$ & $\begin{array}{l}\text { FE.... } \\
\text { CO3... } \\
\text { DS CAL } \\
\text { DEPTH** }\end{array}$ & $\begin{array}{l}337.0 \\
140.0\end{array}$ & $\begin{array}{l}\text { CA..... } \\
\text { SO4 ... } \\
\text { HARD ... }\end{array}$ & $\begin{array}{r}68.0 \\
4.9 \\
308.0\end{array}$ & $\begin{array}{l}\text { MG.... } \\
C L \ldots . \cdots \\
\text { HARD }\end{array}$ & $\begin{array}{l}34.0 \\
10.0\end{array}$ & $\begin{array}{l}\text { NA } \ldots \ldots \\
F \ldots \ldots \\
\text { SP COND }\end{array}$ & $\begin{array}{r}11.0 \\
612.0\end{array}$ & $\begin{array}{l}K_{\ldots} \ldots \ldots \\
N O 3 \ldots \ldots \\
P H \ldots \ldots\end{array}$ & $\begin{array}{r}4.4 \\
.5 \\
7.5\end{array}$ \\
\hline
\end{tabular}

13N-01W-12CBB 1 LAT.- 35-36-56 LONG.- 097-15-50 SEQ.- 1

DATE $(M-D-Y): 06-25-74$

\begin{tabular}{|c|c|c|c|c|c|c|c|c|c|c|}
\hline $\begin{array}{l}C A_{\ldots} \ldots \ldots \\
C L \ldots \ldots \\
P H_{\ldots} \ldots \ldots\end{array}$ & $\begin{array}{r}81.0 \\
17.0 \\
7.6\end{array}$ & $\begin{array}{l}\text { MG } \because \cdots \\
\text { NOS } \because \cdots \\
\text { TEMP } \triangleq C\end{array}$ & $\begin{array}{l}42.0 \\
16.0 \\
19.5\end{array}$ & $\begin{array}{l}\text { NA } \\
\text { DS ROE } \\
\text { DEPTH* }\end{array}$ & $\begin{array}{r}11.0 \\
401.0 \\
160.0\end{array}$ & $\begin{array}{l}\mathrm{HCO} 3 \ldots \\
\text { HARD... }\end{array}$ & $\begin{array}{l}407.0 \\
380.0\end{array}$ & $\begin{array}{l}C O 3 \\
\text { HARD NC }\end{array}$ & 41.0 & SO $_{\text {SP }}$ COND \\
\hline
\end{tabular}

1
I

13N-02W-20BAA 1 LAT.- 35-35-33 LUNG.- 097-26-05 SEQ.- 1 DATE $(M-D-Y): 04-22-54$

\begin{tabular}{|c|c|c|c|c|c|c|c|c|c|c|c|}
\hline $\begin{array}{l}\mathrm{SIOZ}_{\mathrm{H}} \ldots \\
\mathrm{HCO} \ldots \ldots \\
\mathrm{B}_{\ldots} \ldots \ldots \\
\mathrm{PH} \ldots \ldots\end{array}$ & $\begin{array}{r}15.0 \\
352.0 \\
.3 \\
7.4\end{array}$ & 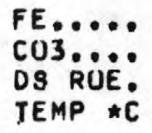 & $\begin{array}{r}306.0 \\
16.5\end{array}$ & $\begin{array}{l}\text { CA.... } \\
\text { SOA \#\#. } \\
\text { DS CAL. } \\
\text { DEPIH:* }\end{array}$ & $\begin{array}{r}64.0 \\
12.0 \\
337.0 \\
100.0\end{array}$ & $\begin{array}{l}\text { MG.... } \\
\text { CL..... } \\
\text { HARD... }\end{array}$ & $\begin{array}{r}37.0 \\
12.0 \\
312.0\end{array}$ & 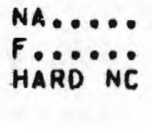 & $\begin{array}{r}11.0 \\
.1 \\
23.0\end{array}$ & $\begin{array}{l}K \ldots \ldots \\
N O 3 \ldots \ldots \\
\text { SP COND }\end{array}$ & $\begin{array}{r}8.0 \\
12.0 \\
581.0\end{array}$ \\
\hline
\end{tabular}


UKLAHUMA CUUNTY

\begin{tabular}{|c|c|c|c|c|c|c|c|c|c|c|c|}
\hline $\begin{array}{r}13 N-03 W=09 B A B \\
\text { UATE }\end{array}$ & $1(M-D=$ & $\begin{array}{l}A:-35-3 \\
111-14=7\end{array}$ & $\begin{array}{l}7-22 \\
4\end{array}$ & LONG $=0$ & $7-31-32$ & $S E Q_{0}-1$ & & & & & \\
\hline 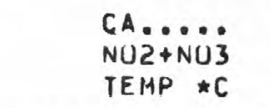 & $\begin{array}{r}55.0 \\
1.9 \\
20.0\end{array}$ & $\begin{array}{l}\text { MG . . . } \\
\text { DS RUE. } \\
\text { DEPTH* }\end{array}$ & $\begin{array}{r}36.0 \\
558.0 \\
147.0\end{array}$ & $\begin{array}{l}\text { NA } \ldots . . . \\
\text { HARO ... }\end{array}$ & $\begin{array}{l}100.0 \\
290.0\end{array}$ & $\begin{array}{l}\text { HCO3 } \\
\text { HARD NC }\end{array}$ & $\begin{array}{r}339.0 \\
7.0\end{array}$ & SUA COONO & $\begin{array}{r}20.0 \\
992.0\end{array}$ & $\begin{array}{l}C L \ldots \ldots \\
P H \ldots \ldots\end{array}$ & $\begin{array}{r}140.0 \\
7.7\end{array}$ \\
\hline
\end{tabular}

$13 N=03 W-20 C C C 1 \quad$ LAT_- 35-34-49 LONG = 097-32-54 SEQ.- 1

DATE $(M-D-Y): 04-13-44$

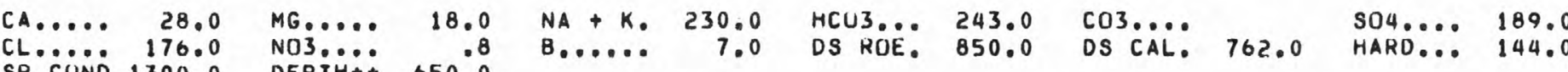
SP CUND 1300.0 DEPTH\# 650.0

13N-03W-28BBD 1 LAT-35-31-27 DATE $(M=D-Y): 05-30-73$

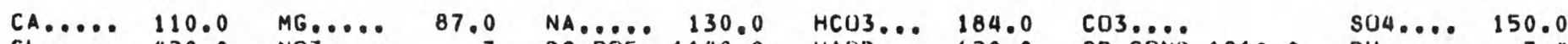

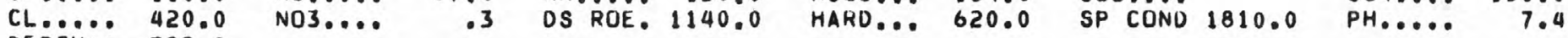
DEPTH* 789.0

13N-03W-29DCA 1 LAT,- 35-34-11 LONG.- 097-32-08 SEQ.-

DATE $(M-D=Y): 07-23-45$

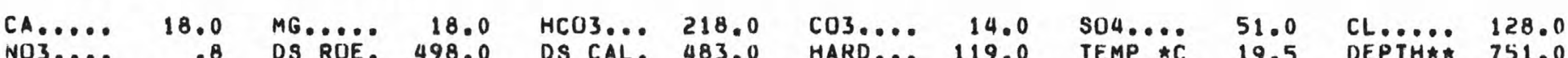

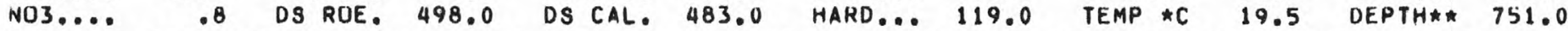

13N=03W-29DDD $1 \quad$ LAT.- 35-33-31 LONG.- 097-31-55 SEQ.- 1 DATE $(M-D-Y): 04-16-43$

\begin{tabular}{|c|c|c|c|c|c|c|c|c|c|c|}
\hline $\begin{array}{l}C A \ldots \ldots \\
C L \ldots \ldots \ldots \\
\text { HARO } \ldots\end{array}$ & $\begin{array}{r}8.5 \\
116.0 \\
40.0\end{array}$ & 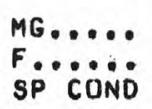 & $\begin{array}{r}4.8 \\
2.6 \\
1160.0\end{array}$ & $\begin{array}{l}N A+K \\
N D 3, \ldots \\
\text { DEPTH* }\end{array}$ & $\begin{array}{r}262.0 \\
.2 \\
790.0\end{array}$ & $\begin{array}{l}\mathrm{HCO} \ldots . . . \\
8 \ldots . .\end{array}$ & $\begin{array}{r}376.0 \\
24.0\end{array}$ & $\begin{array}{l}\text { CO3... } \\
\text { DS ROE. }\end{array}$ & $\begin{array}{r}43.0 \\
651.0\end{array}$ & $\begin{array}{l}\text { SUA } \\
\text { DS CAL. }\end{array}$ \\
\hline
\end{tabular}




\section{DKLAHOMA COUNTY}

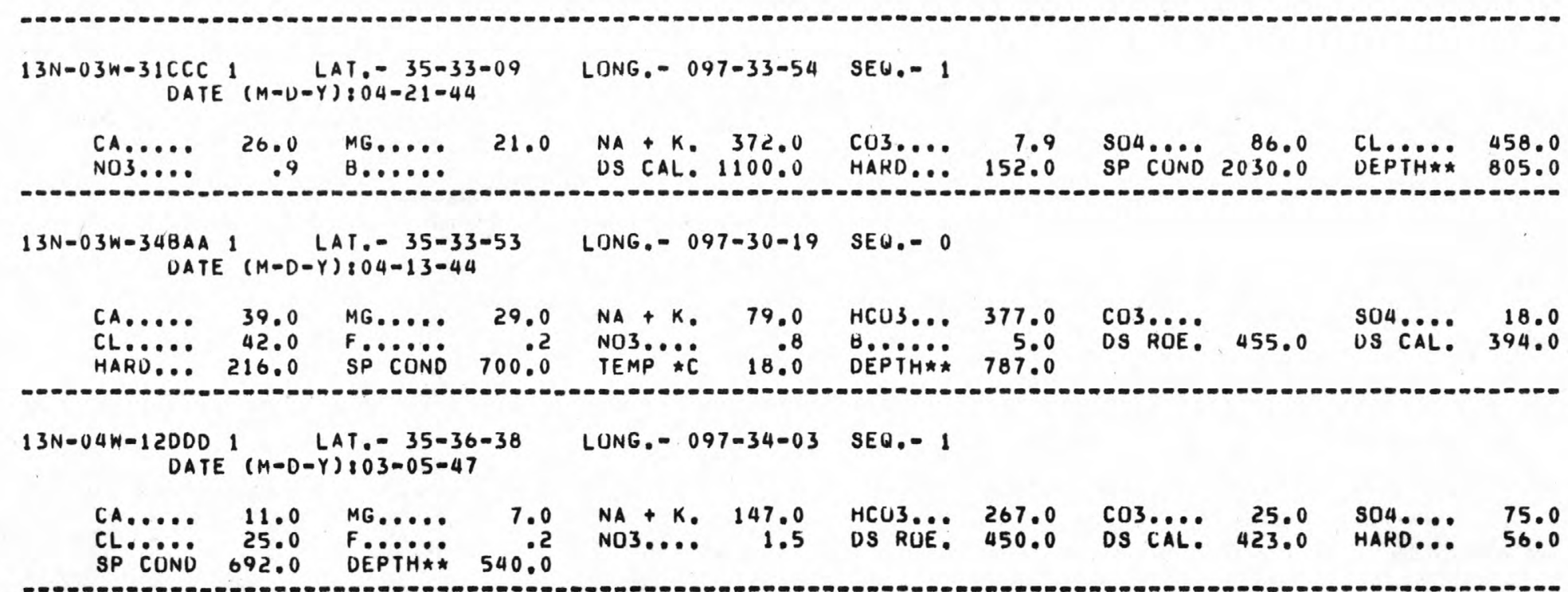

\section{N-04W-22ADA 1 LAT.- 35-35-25 LONG.- 097-36-11 SEQ.- 1 DATE $(M-D-Y): 11-15-74$}

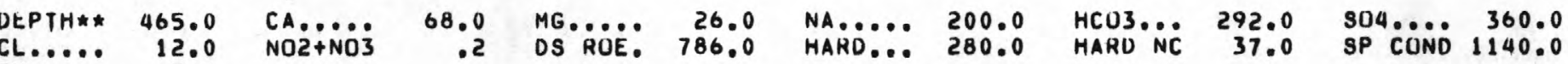
PH.... 7.8

13N-04W-25ABC $1 \quad$ LAT.- 35-34-37 LONG.- 097-34-27 SEQ.DATE $(M-D-Y): 0 B-24-70$

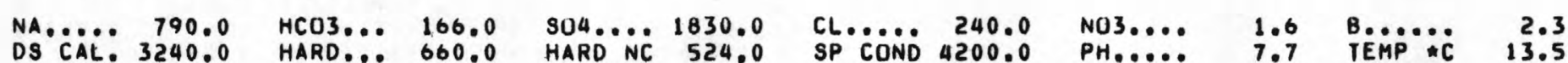
DEPTHA 
UKLAHUMA COUNTY

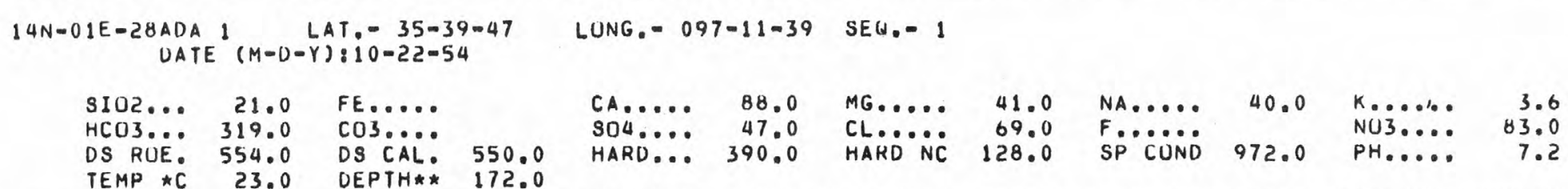

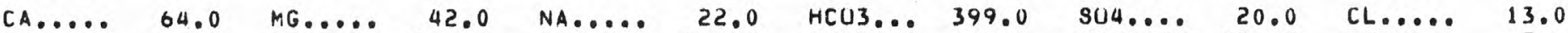
NUZ+NOS $\quad .8$ DS RUE. 367.0 HARD... 330.0 HARD NC 5.0 SP CUND $652.0 \quad$ PH..... OEPTH* 116.0

14N-01W-21BOC 1 LAT.- 35-40-25 LONG.- 097-20-01 SEG.- 1

DATE $(M-0-Y): 07-21-71$

NA..... $420.0 \quad H C O 3 \ldots . .488 .0$

B...

$\cos \ldots$

SO4... $73.0 \quad$ CL..... $330.0 \quad$ NO3.... $\quad .2$ IUN-02W-O3ABB 1 LAT.- 35-41-35 LONG.- 097-23-51 SEQ.- 1 DATE $(M-D-Y): 12-08-74$

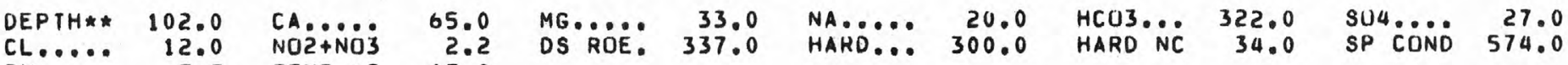
PH...: 7.7 TEMP $\star C \quad 17.0$

14N-02W-09CCD 1 LAT.- 35-41-47 LONG.- 097-25-11 SEG.- 1 DATE $(M-D-Y): 04-22-54$

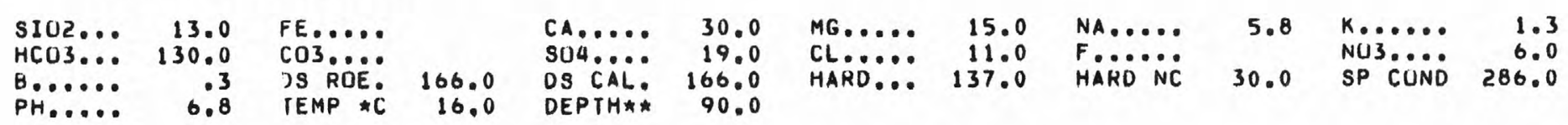




\section{OKLAHUMA COUNTY}

14N-02W-17CBB 1 LAT.- 35-41-20 LONG.- 097-26-31 SEU.- 1

DATE $(M-D-Y): 04-17-44$

\begin{tabular}{|c|c|c|c|c|c|c|c|c|c|c|c|c|}
\hline $\begin{array}{l}C A \ldots \ldots \\
N Q 3 \ldots \ldots \\
D E P T H \star *\end{array}$ & $\begin{array}{r}65.0 \\
5.0 \\
160.0\end{array}$ & $\begin{array}{l}\text { MG ..... } \\
B \ldots \ldots\end{array}$ & $\begin{array}{r}33.0 \\
.1\end{array}$ & $\begin{array}{l}\text { NA } \\
\text { DS }\end{array}$ & $\begin{array}{l}+K . \\
\text { ROE. }\end{array}$ & $\begin{array}{r}18.0 \\
327.0\end{array}$ & $\begin{array}{l}\text { HCU3 } \\
\text { US CAL. }\end{array}$ & $\begin{array}{l}375.0 \\
325.0\end{array}$ & $\begin{array}{l}\text { SO4.... } \\
\text { HARD... }\end{array}$ & $\begin{array}{r}7.0 \\
298.0\end{array}$ & SLP COND & $\begin{array}{r}12.0 \\
582.0\end{array}$ \\
\hline
\end{tabular}

14N-02W-17CDB 1 LAT.- 35-41-01 LONG.- 097-26-10 SEQ.- I

DATE $(M=D-Y): 04-17-44$

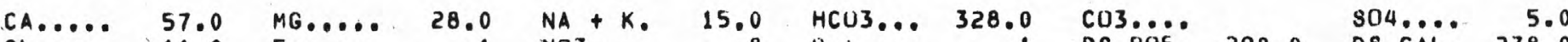

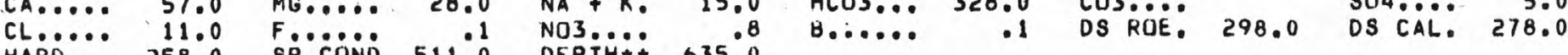
HARD ... 258.0 SP COND 511.0 DEPTH* 635.0

14N-02-23COB 1 LAT.- 35-40-14 LUNG.- 097-23-02 SEQ.-

DATE $(M-D-Y): 11-11=74$

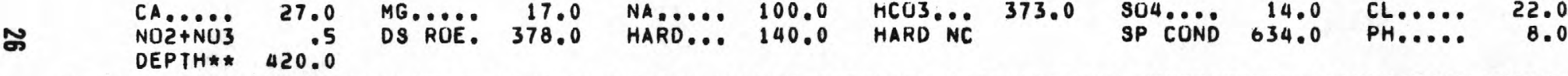

14N-02W-30BAA 1 LAT.- 35-29-55 LONG.- 097-27-10 SEQ.- 1

DATE $(M-D-Y): 12-09-70$

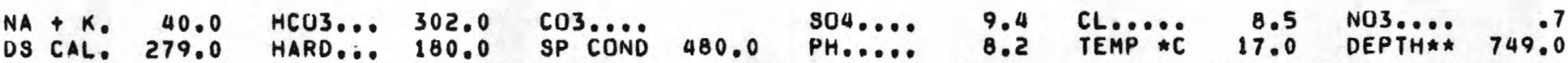

DS CAL. 279.0 HARD... 180.0 SP COND

14N-03W-260AC I LAT.- 35-39-25 LUNG.- 097-28-53 SEQ.- I

OATE $(M-D-Y): 04-15-43$

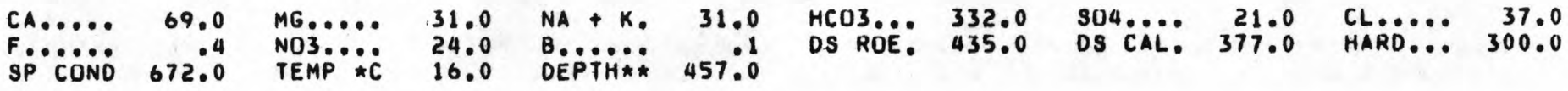


OKLAHIJMA COUNTY

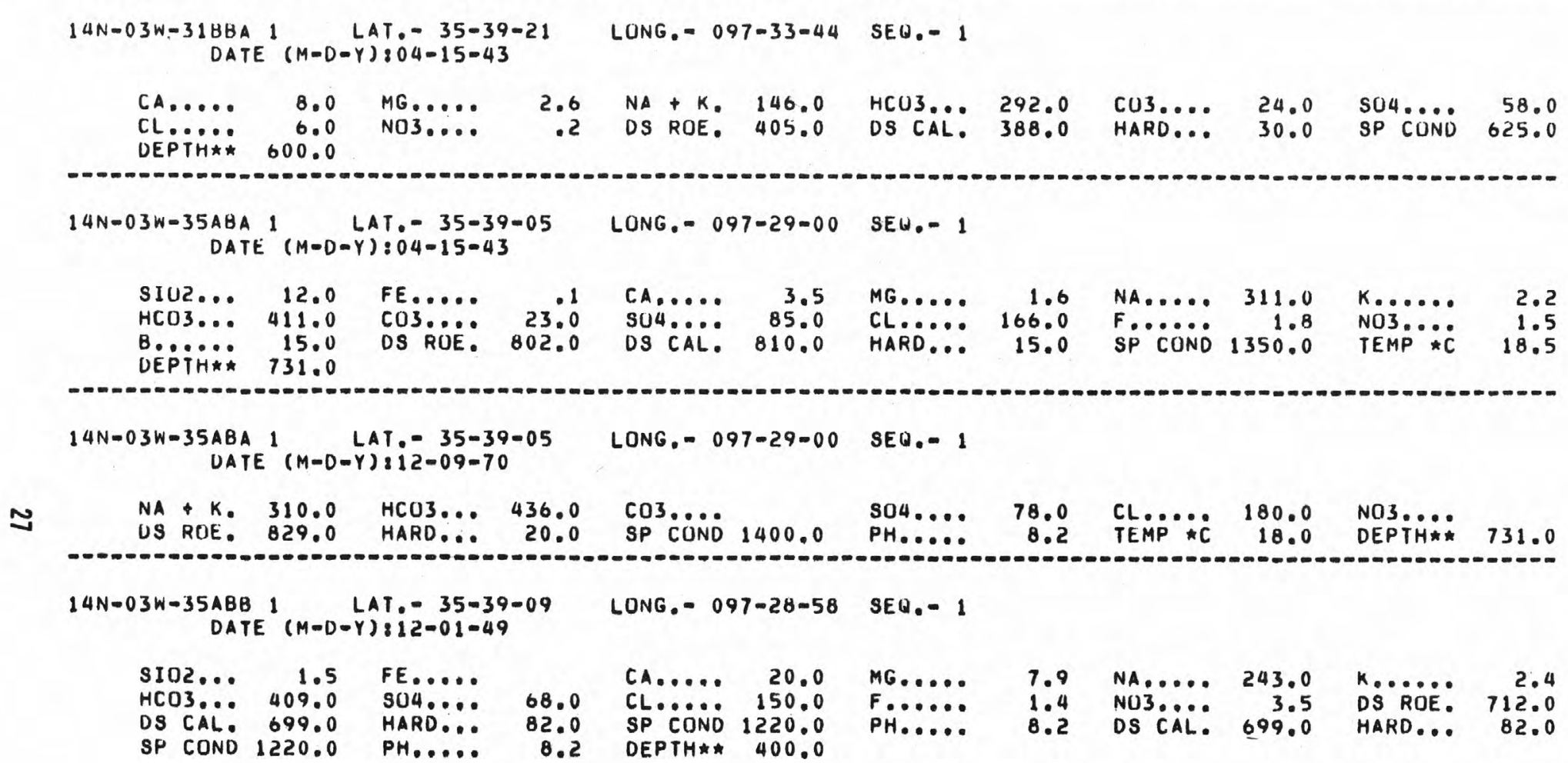


LUGAN COUNTY

\begin{tabular}{|c|c|c|c|c|c|c|c|c|c|c|c|}
\hline \multicolumn{4}{|c|}{$\begin{array}{c}15 N-01 E-18 B C B 1 \quad \text { LAT }=35-46-15 \\
\text { DATE }(M-D=Y) 802-10-71\end{array}$} & \multicolumn{2}{|c|}{ LONG.- $097-14=30$} & \multicolumn{2}{|l|}{ SEU. -1} & \multirow[b]{2}{*}{ 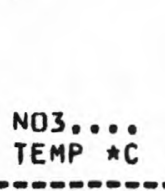 } & \multirow[b]{2}{*}{$\begin{array}{l}66.0 \\
16.0\end{array}$} & \multirow[b]{2}{*}{$\begin{array}{l}\text { DS RUE. } \\
\text { OEPTH** }\end{array}$} & \multirow[b]{2}{*}{$\begin{array}{r}533.0 \\
65.0\end{array}$} \\
\hline $\begin{array}{ll}\text { NA } \\
\text { HARD } \cdots:\end{array}$ & $\begin{array}{r}49.0 \\
350.0\end{array}$ & 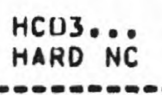 & $\begin{array}{r}372.0 \\
47.0\end{array}$ & SO4 & $\begin{array}{r}35.0 \\
875.0\end{array}$ & CL..... & $\begin{array}{r}46.0 \\
7.6\end{array}$ & & & & \\
\hline \multicolumn{2}{|l|}{$\begin{array}{r}15 N-01 E-26 A A D \perp \\
\text { DATE }\end{array}$} & $\begin{array}{l}\text { AT: }=35-4 \\
812-17-7\end{array}$ & $\begin{array}{l}5-05 \\
4\end{array}$ & LONG.- 09 & $7-09-35$ & SEQ. -1 & & & \multirow[b]{2}{*}{332.0} & \multirow[b]{2}{*}{ SO4 COND } & \\
\hline $\begin{array}{l}\text { DEPTH** } \\
\text { CL..... } \\
\text { PH. }\end{array}$ & $\begin{array}{r}150.0 \\
12.0 \\
7.8\end{array}$ & $\begin{array}{l}\text { CA..... } \\
\text { NOL +NOB } \\
\text { TEMP } \star C\end{array}$ & $\begin{array}{r}56.0 \\
17.2 \\
17.0\end{array}$ & $\begin{array}{l}\text { MG. } \\
\text { DS ROE. }\end{array}$ & $\begin{array}{r}27.0 \\
325.0\end{array}$ & HARD..... & $\begin{array}{r}33.0 \\
250.0\end{array}$ & $\begin{array}{l}\text { HCO3... } \\
\text { HARO NC }\end{array}$ & & & $\begin{array}{r}8.4 \\
543.0\end{array}$ \\
\hline \multicolumn{2}{|c|}{$\begin{array}{l}15 N-01 W-20 D^{\prime} D^{1} \\
\text { DATE }(M-D\end{array}$} & AT: $=\begin{array}{r}35-4 \\
\text { ): }\end{array}$ & $\begin{array}{l}5-14 \\
4\end{array}$ & \multicolumn{2}{|c|}{ LONG.- $097-19-21$} & \multicolumn{2}{|l|}{ SEQ.- 1} & \multirow[b]{2}{*}{$\begin{array}{l}\text { HCO3,... } \\
\text { SP COND }\end{array}$} & \multirow[b]{2}{*}{$\begin{array}{l}357.0 \\
658.0\end{array}$} & \multirow[b]{2}{*}{$\begin{array}{l}\text { SO4.... } \\
\text { PH..... }\end{array}$} & \multirow[b]{2}{*}{$\begin{array}{r}67.0 \\
6.0\end{array}$} \\
\hline $\begin{array}{l}\text { DEPTH** } \\
\text { CL } \because \because \cdots \\
\text { TEMP }\end{array}$ & $\begin{array}{r}31.0 \\
6.6 \\
16.5\end{array}$ & CAOZ+NOB & $\begin{array}{r}95.0 \\
.2\end{array}$ & MG ${ }_{\text {DSOE: }}$ & $\begin{array}{r}23.0 \\
396.0\end{array}$ & NAPOPO & $\begin{array}{l}20.0 \\
39.0\end{array}$ & & & & \\
\hline \multicolumn{2}{|c|}{$\begin{array}{r}15 \mathrm{~N}-02 W-03 A A B B^{1} \\
\text { DATE }(M-D\end{array}$} & $\left\{\begin{array}{l}1,-35=4 \\
: 06-06-7\end{array}\right.$ & $6-50$ & LONG.- 09 & $7-23-45$ & SEQ.- 1 & & & \multirow[b]{2}{*}{35.0} & \multirow[b]{2}{*}{ SOA COND } & \multirow[b]{2}{*}{$\begin{array}{r}20.0 \\
682.0\end{array}$} \\
\hline $\begin{array}{l}C A \ldots \ldots \\
C L \ldots \ldots \\
P H \ldots \ldots\end{array}$ & $\begin{array}{r}78.0 \\
24.0 \\
7.5 \\
-\end{array}$ & $\begin{array}{l}M G \ldots \ldots \\
N Q 2+N O \dot{3} \\
\text { TEMP } A C\end{array}$ & $\begin{array}{r}33.0 \\
6.4 \\
18.5 \\
\end{array}$ & $\begin{array}{l}\text { NA. } \\
\text { OS ROE: } \\
\text { DEPTHA }\end{array}$ & $\begin{array}{r}22.0 \\
393.0 \\
66.0\end{array}$ & $\begin{array}{l}\text { HCO3... } \\
\text { HARD... }\end{array}$ & $\begin{array}{l}360.0 \\
330.0\end{array}$ & CO3...̈̈ & & & \\
\hline $\begin{array}{r}15 N=03 W=11 B A B \\
\text { DATE }\end{array}$ & $E^{1}(M=0$ & \multicolumn{2}{|c|}{$\begin{array}{l}\text { LAT: }=35-47-40 \\
\text { Y):II-19-74 }\end{array}$} & \multicolumn{2}{|c|}{ LONG.- $097-29-30$} & \multicolumn{2}{|l|}{$\operatorname{SEQ} \cdot-1$} & & & & \\
\hline NOZZ+NÖB & $\begin{array}{r}170.0 \\
63.0\end{array}$ & $\begin{array}{l}\text { MG...... } \\
\text { DS ROE. }\end{array}$ & $\begin{array}{r}50.0 \\
1120.0\end{array}$ & $\begin{array}{l}\text { NA...... } \\
\text { HARO... }\end{array}$ & $\begin{array}{l}100.0 \\
630.0\end{array}$ & HCO3. & $\begin{array}{l}379.0 \\
320.0\end{array}$ & SP & $\begin{array}{r}140.0 \\
1590.0\end{array}$ & CLEPTH*:..: & $\begin{array}{r}98.0 \\
100.0\end{array}$ \\
\hline
\end{tabular}


LUGAN CUUNTY

15N-03W-33ACA 1 LAT.- 35-44-07 LONG.-097-31-07 SEU.DATE $(M-D-Y): 11-13-74$

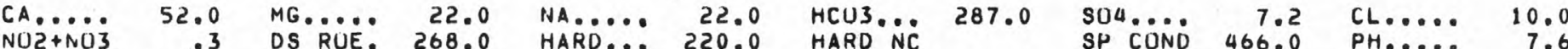

15N-04W-24BBC 1 LAT. - 35-45-55 LONG.- 097-34-59 SEQ.- 1

DATE $(M=0-Y): 06-28-74$

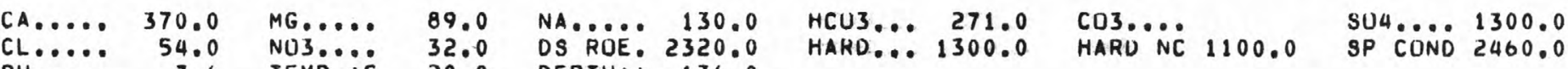

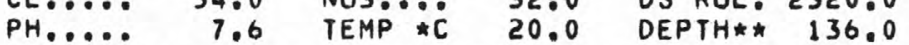

15N-04W-29ABA 1 LAT.- 35-45-16 LUNG. = 097-38-47 SEQ.-

DATE $(M-D-Y): 06-21-74$

ญ

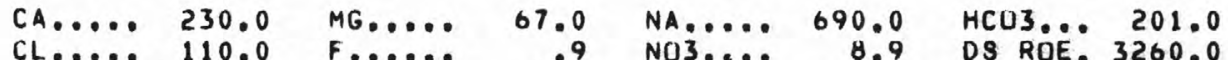

SP COND 500

....

.9

NOS

8.9

S ROE 3260.0

DEPTH** 150.0

$16 N=01 E-08 C D D 1 \quad$ LAT.- 35-52-05 LONG.- 097-13-24 SEQ.-

DATE $(M-D-Y): 11-21-74$

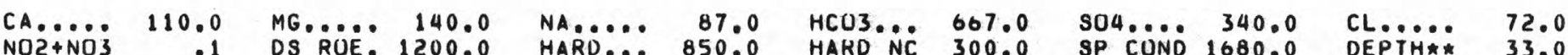

NO2+NO3 1 DS RUE. 1200.0 HARD... 850.0 HAKD NC 300.0 SP CUNO 1680.0 DEPTH** 33.0

16N-01W-29AAA I LAT,-35-49-16 LONG.- 097-19-12 SEQ.- 1

DATE $(M-D-Y): 12-12-74$

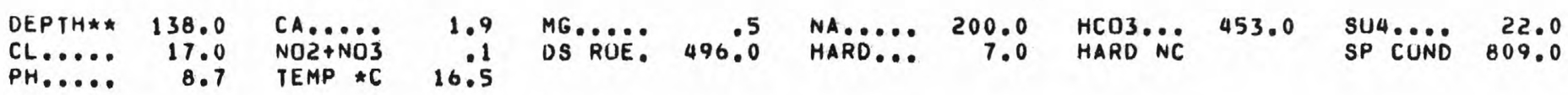


LOGAN CUUNTY

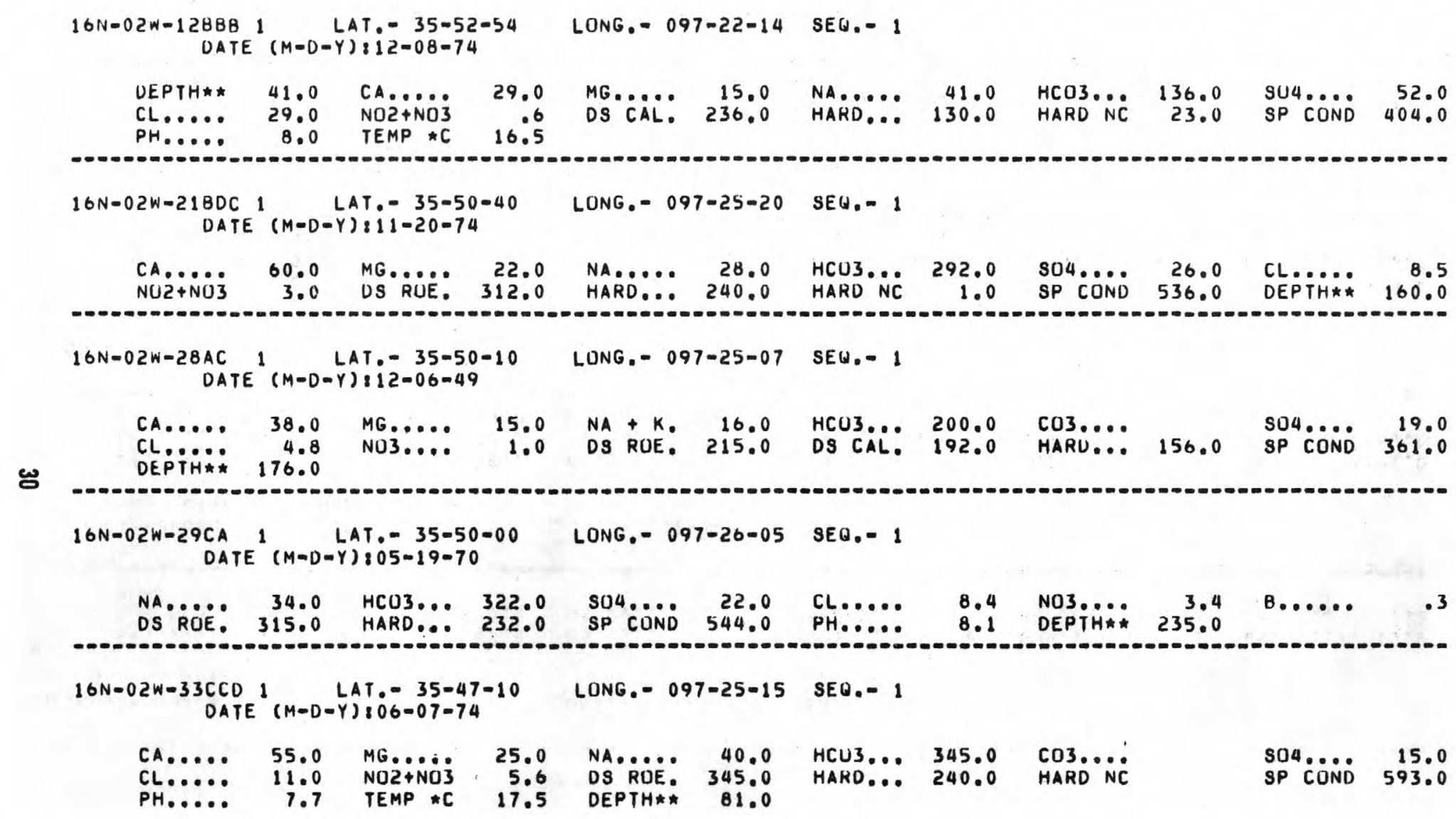




\section{LUGAN CUUNTY}

16N-03W-040DB $1 \quad$ LAT.- 35-53-16 LUNG.- 097-31-05 SEU.- 1

DATE $(M-D-Y): 05-08-74$

\begin{tabular}{|c|c|c|c|c|c|c|c|c|c|c|c|}
\hline $\begin{array}{l}C A \ldots \ldots \\
C L \ldots \ldots \\
P H \ldots \ldots\end{array}$ & $\begin{array}{r}64.0 \\
10.0 \\
7.7\end{array}$ & $\begin{array}{l}M G \ldots \ldots \\
N O 2+N O 3 \\
T E M P \star C\end{array}$ & $\begin{array}{r}22.0 \\
6.0 \\
19.5\end{array}$ & $\begin{array}{l}\text { NA } \ldots . . \\
\text { DS RUE. } \\
\text { DEPTH* }\end{array}$ & $\begin{array}{r}25.0 \\
336.0 \\
125.0\end{array}$ & $\begin{array}{l}\text { HCU3... } \\
\text { HARD... }\end{array}$ & $\begin{array}{l}304.0 \\
250.0\end{array}$ & $\begin{array}{l}\text { CO3.... } \\
\text { HARD NC }\end{array}$ & 1.0 & $\begin{array}{l}\text { SU4 } \\
\text { SP COND }\end{array}$ & $\begin{array}{r}14.0 \\
562.0\end{array}$ \\
\hline
\end{tabular}

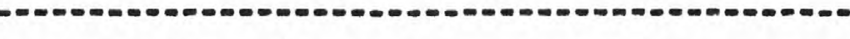

16N-03W-1100D 1 LAT - 35-52-25 LUNG.- 097-28-57 SEQ.- 1

DATE $(M-D-Y): 05-22-74$

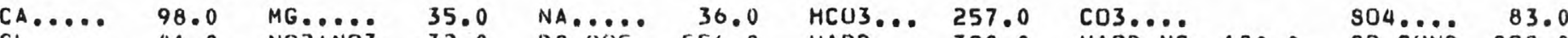

CL..... 41.0 NOZ+NO3 32.0 OS RUE. S56.0 HARD... 390.0 HARD NC 180.0 SP CUND B98.0

PH.... 7.8 TEMP $\star C \quad 17.0$ DEPTH* 112.0

16N-03W-18ADD 1 LAT.-35-51-48 LONG.- 097-33-01 SEQ.- 1

DATE $(M-O-Y): 11=06-70$

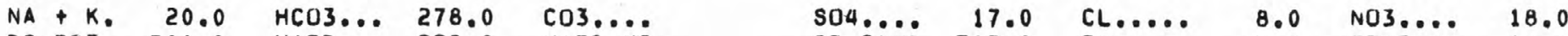

DS RUE, 301.0 HARD... 228.0 HARD NC SP CUND 502.0 PH.... 8.1 TEMP $\star$. 16.0
DEPTH* 125.0

16N-03W-25DAD 1 LAT.- 35-49-50 LONG.- 097-27-40 SEQ.- 1

DATE $(M-D-Y): 11-06-70$

\begin{tabular}{|c|c|c|c|c|c|c|c|c|c|c|}
\hline $\begin{array}{l}\text { NA }+K \\
\text { DS ROE. } \\
\text { OEPTH** }\end{array}$ & $\begin{array}{r}43.0 \\
378.0 \\
200.0\end{array}$ & $\begin{array}{l}\text { HCO3... } \\
\text { HARD... }\end{array}$ & $\begin{array}{l}346.0 \\
262.0\end{array}$ & $\begin{array}{l}\text { CO3... } \\
\text { HARD }\end{array}$ & $\begin{array}{l}\text { SO4 } \\
\text { SP COND }\end{array}$ & $\begin{array}{r}21.0 \\
647.0\end{array}$ & $\begin{array}{l}C L \ldots \ldots \\
P H \ldots \ldots\end{array}$ & $\begin{array}{r}30.0 \\
8.0\end{array}$ & $\begin{array}{l}\text { NO3 } \cdots \\
\text { TEMP } * C\end{array}$ & $\begin{array}{r}8.7 \\
18.0\end{array}$ \\
\hline
\end{tabular}

DATE $(M-D-Y): i 1=19-74$

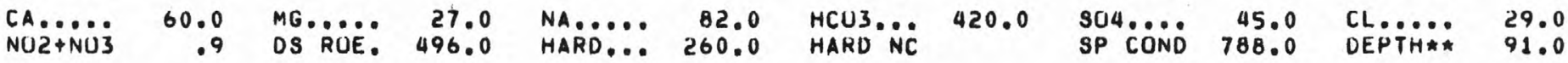


LUGAN COUNTY

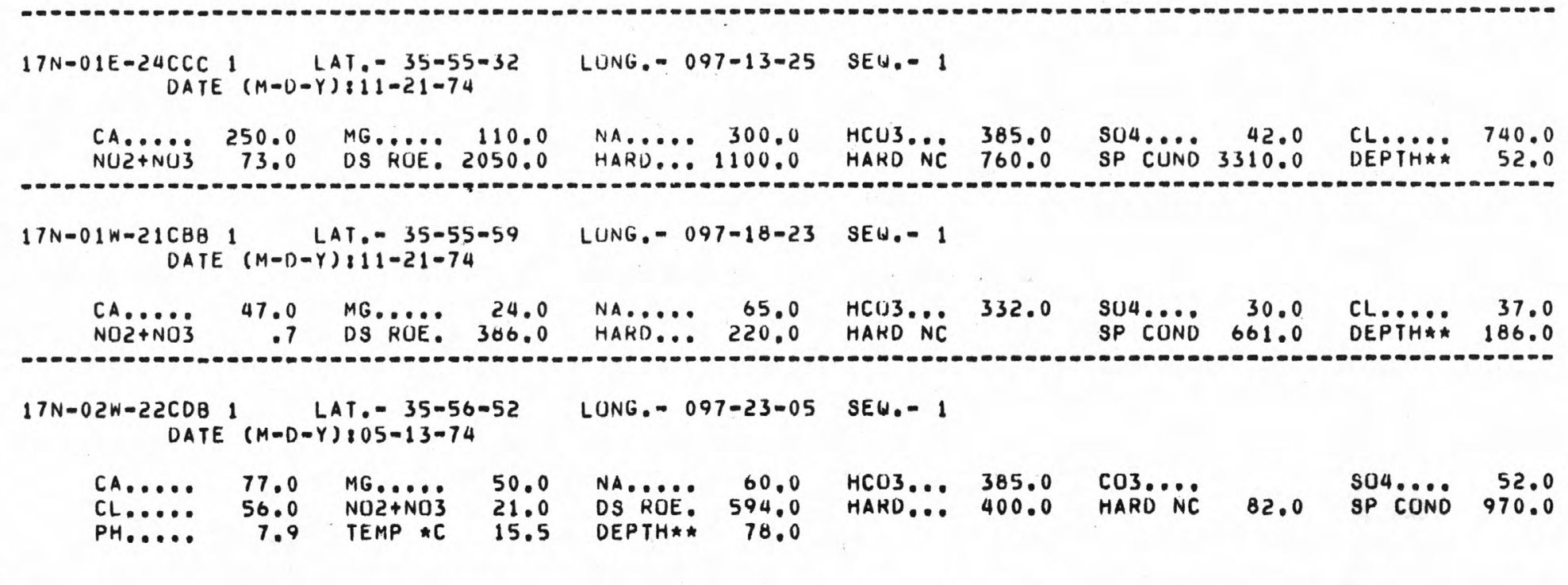


